\title{
Optimal Production Strategies for the Development of Mature Oil Fields through Polymer Flooding
}

\author{
Demian J. Presser, Vanina G. Cafaro, and Diego C. Cafaro*
}

Cite This: https://doi.org/10.1021/acs.iecr.1c00803

Read Online

ABSTRACT: Polymer flooding is one of the most extensively applied techniques for enhanced oil recovery (EOR) that relies on increasing the injected fluid viscosity by adding polymers in different concentrations. The aim is to improve the mobility ratio between the oil and the displacing fluid, reducing channeling effects and boosting sweep efficiency. In this work, a mixed-integer nonlinear optimization approach is developed in order to address production planning decisions, also assessing the convenience of polymer flooding in EOR projects on screening stages. This decision-making tool allows establishing optimal polymer injection strategies, setting fluid injection rates and polymer concentrations, selecting wells, and scheduling slug change operations over the planning horizon, with the aim of maximizing the net present value of the project. We propose a

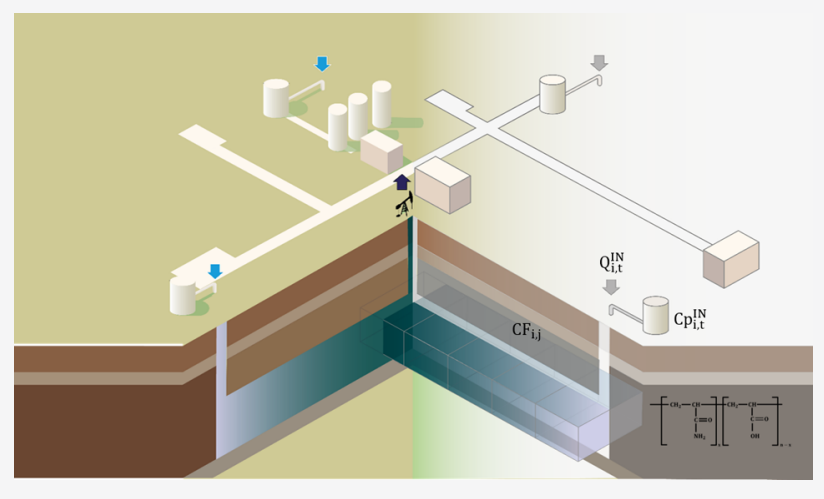
novel formulation combining transport phenomenon models, well productivity forecast, and geometric abstraction of the reservoir in order to address both technical and economic decisions with low computational effort. Results show that optimized strategies may outperform typical operations by up to $17 \%$ and can be found in shorter central processing unit times in comparison to traditional reservoir simulation approaches. The model has been used to tackle problems with up to 7 wells and can be tuned to different reservoir conditions.

\section{INTRODUCTION}

Despite the great advances in terms of renewable energies in the last few years, fossil fuels remain as the major source of energy over the world. Moreover, a sustained demand for oil and gas is projected for the next 30 years, ${ }^{1}$ leading to even greater requirements and consistent response from the industry. Enhanced oil recovery (EOR) techniques continue to increase in relevance due to their capacity of recovering large amounts of hydrocarbons that stand on conventional reservoirs after primary and secondary production stages. EOR comprises an extensive set of advanced methods aimed to take advantage of substantial modifications of physical and chemical properties of the trapped hydrocarbons in order to improve recovery efficiency on apparently depleted mature oil fields. While oil field discoveries per year decrease steadily, the development of EOR techniques is becoming more and more crucial.

Polymer flooding stands as one of the most important EOR techniques consisting of adding soluble polymers with high molecular weight to the injection water. The polymer solution holds a higher viscosity that boosts the mobility ratio between the injected fluid and the remaining crude oil, stabilizes the displacing fronts, reduces channeling effects, and, hence, leads to a better overall sweep efficiency. ${ }^{2-5}$ The polymer flooding process (Figure 1) targets oil reservoirs that contain oil with relatively high viscosities (from 50 to $5000 \mathrm{cp}$ ) and densities (from 12 to 22.3 API). ${ }^{6}$ This aspect makes it a promising method for the exploitation of many mature viscous-oil reservoirs over the world. Nevertheless, the successful implementation of polymer flooding requires a thorough understanding of the influence of design parameters on its performance. ${ }^{7}$ Even though chemical-based EOR initiatives like polymer flooding are frequently considered as a natural step after the waterflooding stage in mature oil fields, several investments, reservoir characterization, pilot tests, and productivity assessments must be carried out. These tasks might be highly expensive, thus requiring agile decision-making tools that allow identification of suitable stimulation strategies and estimate their economic potential before heavy capital expenditures are made or further research is performed. Furthermore, tools that provide accurate predictions through smart production strategies can serve as guidelines for the real scenario and have become more and more necessary for the modern oil and gas industry. ${ }^{8}$

Received: March 2, 2021

Revised: May 23, 2021

Accepted: June 25, 2021 


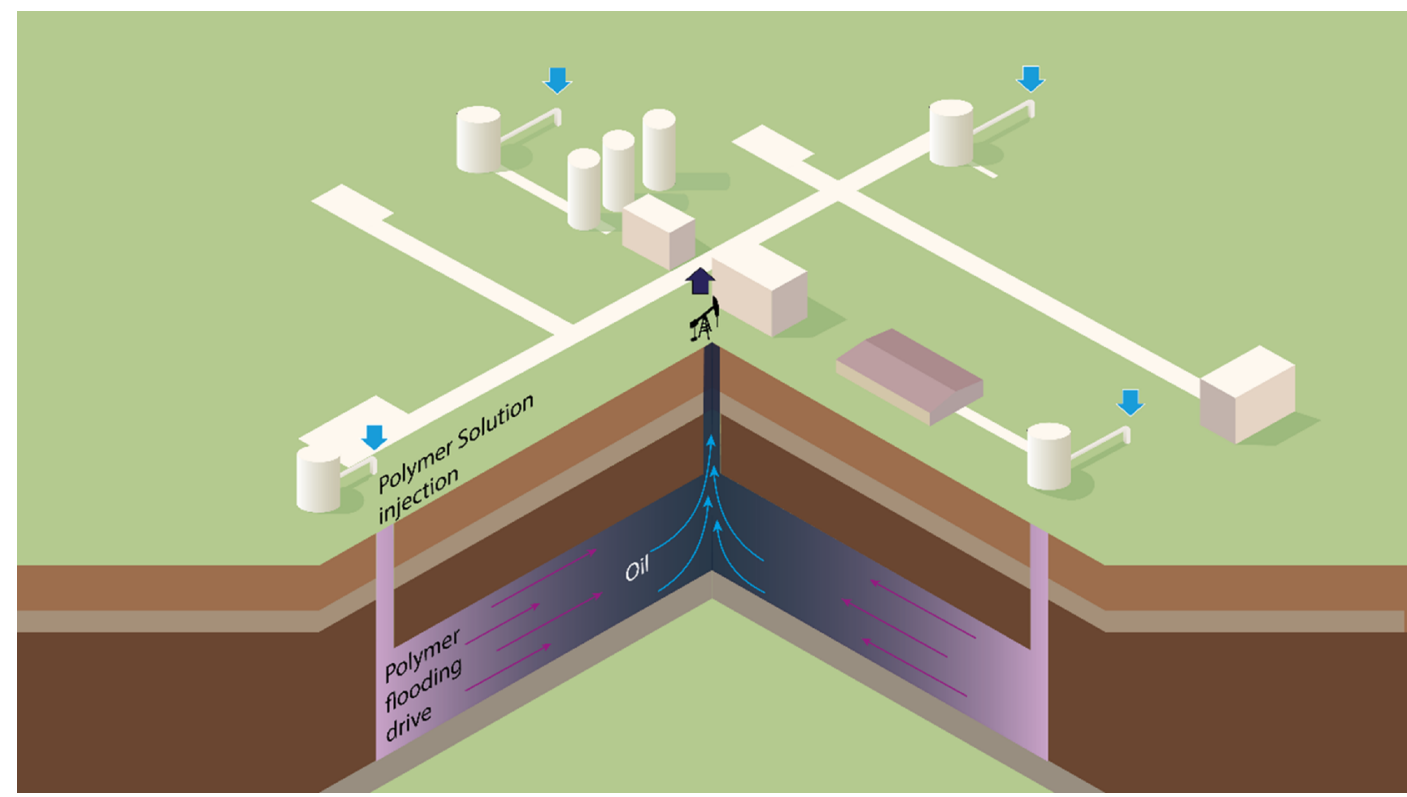

Figure 1. Production scheme of enhanced oil recovery (EOR) through polymer flooding.

Technical and economic optimization of polymer flooding strategies has been the focus of several research works. Wang et al. ${ }^{9}$ reviewed the most important aspects in the design of polymer flooding projects based on the Daqing oil fields in China, drawing important conclusions about their overall effectiveness depending on the polymer solution viscosity, slug size, injectivity, injection rates, and economics. Horowitz et al. ${ }^{10}$ presented a global optimization algorithm considering uncertainty to determine optimal production strategies. The authors made use of design-of-experiment methods to determine key simulation scenarios and formulate a surrogate model that replaces more complex reservoir simulations. After defining a forecast function, a Kriging $\operatorname{model}^{11}$ was used to suggest optimal polymer concentration, injection start time, and slug duration on a given set of injection wells. Since the first step of this tool is based on reservoir simulation, each functional evaluation requires a complete simulation run, yielding a highly expensive computational method. Li et al. ${ }^{12}$ and Lei et al. ${ }^{13}$ used genetic algorithms to determine optimal injection strategies for polymer flooding, assuming reservoir model equations as a black box. Genetic algorithms are capable of finding the global optimum on a theoretical sense but at the expense of requiring thousands of reservoir simulation runs of extensive models. Lei et al. ${ }^{14}$ proposed an iterative dynamic programming model to solve the polymer flooding optimal control problem, defining the slug volume, polymer concentration, and flooding completion time. Results are promising, showing the importance of a smart operation strategy on polymer flooding projects.

AlSofi and Blunt ${ }^{7}$ used streamline-based simulations applied to a thousand of design sets in order to determine optimal concentrations, slug run durations, and starting times, based on technical and economic criteria to maximize the net present value (NPV) of polymer flooding projects. The authors concluded that starting times are trivial decisions since their model predicts that the sooner is always the better. However, the NPV shows strong sensitivity to polymer concentration and slug sizes. Decision variables related to oil field design such as well selection and operation mode are not considered in that work. Ekkawong et al. ${ }^{15}$ presented a new multiobjective optimization framework based on genetic algorithms in order to address optimal concentrations, slug sizes, and injection rate allocation for polymer flooding. They showed interesting results in terms of solving the trade-off between cumulative oil production and polymer efficiency. However, the authors highlighted that the linkage between rate optimization via streamline methods and polymer fluid optimization via genetic algorithms is rather weak. Similarly, Janiga et al. ${ }^{16}$ presented a wide set of nature-inspired methods such as a bee colony, a firefly algorithm, and a gray wolf optimizer, among others, and combined them with reservoir simulations to establish optimal control variables in polymer flooding. Physical constraints are implicitly considered by an Eclipse reservoir simulator ${ }^{17}$ for EOR.

In general terms, capacitance resistance models (CRM) are the standard for predicting flows in mature oil and gas fields. They stand as electric circuit analog representations aimed to estimate the way in which injected fluids are distributed through the subsurface toward producing wells. Several applications of these models can be found in the literature, which can be classified into waterflooding, ${ }^{18-22}$ gas flooding, ${ }^{23}$ and EOR strategies. ${ }^{24-26}$ Holanda et al. ${ }^{27}$ carried out an indepth literature review of CRM used in reservoir characterization and performance forecasting. In this comprehensive work, the authors highlighted promising aspects of CRM implementations for EOR projects, also emphasizing the need for accurate fractional flow models to predict the flow composition as an encouraging field for future research. In this direction, Cao et al. ${ }^{28}$ presented an original technique for oil production forecast by combining the Koval theory ${ }^{29}$ with CRM.

The Koval theory is an extensively used representation that has been developed to predict the oil production on miscible displacement techniques. The theory is stated as a robust model with a much broader scope than the one originally planned for it. $^{30}$ For instance, Mollaei and Delshad ${ }^{31}$ proposed a general isothermal enhanced oil recovery production forecast tool drawn from the analytical interpretation of the Koval theory. 
Similarly, Jain and $\mathrm{Lake}^{32}$ relied on the Koval theory to successfully represent EOR displacement phenomena, while Farajzadeh et al. $^{33}$ provided valuable insights into mobility control design for polymer flooding making use of the modified Koval theory. More recently, Salazar and Lake ${ }^{34}$ developed a comprehensive theoretical framework for the physical interpretation of Koval model parameters. The authors carried out a deep analysis on reservoir heterogeneity and Koval factors based on the results of numerical simulations.

Regarding specific optimization approaches based on simplified reservoir modeling, Eshraghi et al. ${ }^{25}$ made use of $\mathrm{CRM}$ and semiempirical hyperbolic fractional flow functions to optimize $\mathrm{CO}_{2}$-EOR projects using heuristic methods. In turn, Presser et al. ${ }^{35}$ combined CRM with data-driven fractional flow functions yielding a mixed-integer nonlinear mathematical programming (MINLP) formulation that determines optimal operation strategies for EOR with $\mathrm{CO}_{2}$. Results suggest that the optimization approach could be extended to different EOR techniques, also accounting for uncertainty in oil prices and well productivity.

This work presents a novel mathematical programming approach aiming to make optimal decisions on important aspects of mature oil field exploitation. The proposed model is focused on polymer flooding production strategies in order to maximize the net present value over a long-term planning horizon. This tool is planned to serve as an accurate evaluation step before carrying out more in-depth studies and, additionally, to compare against alternative EOR techniques identified during screening stages. The optimization tool integrates two models of proven efficiency for oil production forecast: capacitance resistance modeling ${ }^{36}$ and the Koval theory. ${ }^{29}$ Moreover, we develop a novel geometric abstraction of the reservoir to represent the drainage volumes around the wells in the field. While reservoir simulations are very common in the oil and gas industry, integrating these models within optimization frameworks is still very challenging. In this work, we demonstrate that novel abstractions of the time and space domains may certainly help. Several technical aspects of polymer flooding such as polymer adsorption, permeability reduction, and mobility performance are all taken into account in the optimization model, solving different trade-offs such as ultimate recovery vs production rates or polymer concentration (sweep efficiency) vs adsorption (permeability reduction). Finally, two illustrative study cases based on real-world data are presented, solved, and discussed.

1.1. Novel Contributions of This Work. The model presented in this work provides a general optimization framework to address the polymer flooding planning problem. By means of this tool, we are able to determine the optimal values of several decision variables that are not accounted for altogether in previous contributions. Previous approaches usually consider a limited subset of operating variables such as polymer concentration, injection rates, and/or slug sizes, with some of these works also addressing the end time of the project as a decision variable. Instead, our approach integrates well selection and timing decisions, which provide valuable guidelines to reservoir engineers. Moreover, most of previous works are based on reservoir simulation (simulation sampling), which permits inclusion of more detailed aspects of the subsurface but is certainly very time-consuming. Furthermore, results from simulation-based tools are highly dependent on the number of trials, from which it is unlikely to find the actual optimal recovery strategy. Although space-time reservoir behavior is captured by simplified correlations and geometric abstractions in this work, our assessment tool can significantly reduce the domain over which decision-makers might develop more in-depth studies, pointing out a reduced number of candidate wells and the reservoir volume to be simulated, as well as tighter bounds on operating variables. Table S1 in the Supporting Information presents a brief comparison among the most relevant optimization models proposed in the literature, $7,10,14,15,37$ highlighting the novel features of the current work.

\section{PROBLEM DEFINITION}

The design of polymer flooding strategies involves a series of key decisions that must be addressed in order to optimize the incremental production of oil in mature fields. Critical aspects highlighted in the literature include slug size, polymer concentration and salinity (affecting slug viscosity and mobility), flooding starting time, injection rates, and well operation (production or injection). In this work, some of the most important decisions are addressed by the optimization framework. For instance, the selection of production wells from a set of existing candidates might depend on the remaining oil over its drainage area, connectivity with injection wells, and reservoir characteristics. In turn, operational decisions may depend on even more technical aspects of the reservoir response to polymers. Injecting polymer solutions (instead of freshwater) at high concentrations intensifies the adsorption phenomena and loss of injectivity. While adsorption causes polymer losses and permeability reduction, injectivity issues may require slowing down of injection rates to keep the formation safety. A thorough understanding of these phenomena could yield better recovery factors, ultimate profits, and additional insights into the problem itself.

The optimal polymer flooding problem addressed in this work can be stated as follows: given (i) an oil reservoir to develop by polymer flooding, (ii) a set of existing wells labeled according to their potential operation mode (injection/ production), (iii) the characterization of sweep volumes and connectivity between every pair of wells, and (iv) a multiperiod planning horizon, the aim is to determine (a) the optimal set of wells to be operated during the project, (b) their operating time windows over the time horizon, (c) the fluid injection rate at each well, in every period, and (d) the polymer concentration and the size of the slug to be injected at every period, in order to maximize the net present value of the project. Technical and economic constraints need to be taken into account. The first ones have to do with flow balances, adsorption phenomena, permeability reduction, viscosity calculation, mobility ratio assessment, flow composition, and connectivity between the wells. Economic equations refer to cost allocation, time value of cash flows, investments, and operating costs.

2.1. Model Assumptions. The model presented in this work is based on the following assumptions:

(a) The field has been producing under waterflooding strategies for a considerable time. From that, we assume that critical information related to the flow distribution (well connectivity), response times between potential injectors and producers, estimated sweep volumes between wells, and petrophysical properties such as water saturation, oil viscosity, irreducible water saturation, residual oil saturation, and inaccessible pore volume of the reservoir are available. 
(b) Existing and/or potential wells to be operated, their location, and possible operation modes (injector/ producer) are given.

(c) Polymer adsorption and reservoir permeability reduction effects are assumed to be computable based on the polymer concentration of the flow across each control volume. According to practice, both processes are considered permanent (irreversible) effects, usually quantified from the Langmuir isothermal function. ${ }^{4}$ Shear thinning effects are neglected. ${ }^{38}$

(d) Sweep volumes between every pair of wells are geometrically abstracted and discretized in this work, establishing a sequence of control volumes or blocks between every injector-producer pair of wells.

(e) In line with the CRM, ${ }^{36}$ the linear velocity of the flow between a pair of wells (and therefore the response time) is assumed to remain constant regardless of the injection rate. Given that the cross-sectional areas of the control volumes are also fixed, we assume that larger injection rates just make the flow reach larger interstitial spaces across the control volumes in the path. In addition, a totally unidirectional flow is assumed.

(f) Polymer diffusion effects are negligible in comparison to concentration gradients due to the movement of the slugs of polymer solution along the sweep volumes or paths. ${ }^{14}$

(g) Flows along paths between different pairs of wells are considered noninteracting. In line with the CRM, we assume that the intersection between the paths connecting two pairs of wells is negligible (see Figure $S 1$ in the Supporting Information). If this assumption does not apply, then artificial (intermediate) nodes and shared paths can be included in the model, but this is beyond the scope of this work.

(h) We assume two-phase (polymer solution and oil) incompressible flows according to the premises of the embedded models. ${ }^{29,36}$ Further details on this assumption can be found in Cao et al., ${ }^{28}$ Zhong et al., ${ }^{40}$ and Koval. $^{29}$

(i) Connectivity coefficients ${ }^{36}$ between every pair of wells remain constant during the planning horizon.

(j) Bottomhole pressures (BHP) in producing wells are assumed to be constant during the time horizon (typically one year). BHP variations are often omitted in CRM models when accounting for relatively short time horizons. $^{22,23}$

Assumption (a) is required for the appropriate parametrization of the petrophysical models. The CRM, in particular, requires a substantial production history to establish the flow scheme between injectors and producers. We assume that this information becomes available from the previous exploitation of the field. Another important assumption is that both polymer adsorption and reservoir permeability reduction phenomena can be predicted by knowing the amount of the polymer in the slug and at each point of the reservoir (assumption (c)). While the adsorption phenomenon makes the displacing fluid reduce its polymer concentration, it also contributes to the reduction of permeability due to porosity blockage, and such permeability reduction impacts on injectivity (maximum injection rate). We assume that these effects are strictly associated with polymer concentration and slug flow over time. While some other variables like water salinity, injection rates, and pressure may affect these phenomena, there is general agreement in the literature to consider these effects as a function of the concentration when most of the other variables are under control within relatively limited ranges. ${ }^{13}$

Assumption (d) is related to the subsurface representation. The sweep volume in every path is discretized in blocks, and petrophysical properties are averaged over the three dimensions of every block. Discontinuity in linking sections between adjacent blocks with heterogeneous anisotropic properties might not be totally accurate, but it is acceptable if properties do not vary significantly and volume discretization is fine enough. In turn, assumption (e) states that the linear fluid velocity remains constant along each path, regardless of the flow rate. In other words, a larger flow rate results in a denser set of streamlines (pores) being accessible by the solvent, with no change in the time it takes for the slug to move from one block to the following. On the contrary, lower injection rates reduce the number of sweep streamlines through each control volume, producing less solvent saturation over time (Figure 2). Note

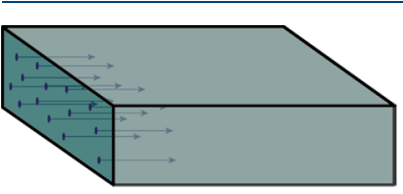

(a)

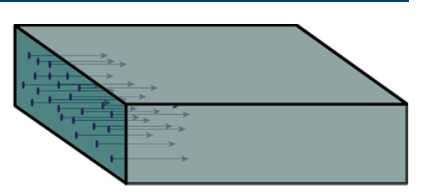

(b)
Figure 2. Constant velocity assumption: lower (a) and higher (b) flow rates through the porous media.

that the slug flow is assumed to be unidirectional, with fixed inlet and outlet sections for every block in the path. All of these are consistent with the CRM model, where the time constant parameter (i.e., the response time to injection signals) is stated as independent of the flow rate.

Other hypotheses assumed by the physical models that are integrated to our approach (namely, the Koval theory and CRM) are also considered in this work. More details on these assumptions can be found in $\mathrm{Koval}^{29}$ and Yousef et al., ${ }^{36}$ respectively.

\section{MODELING FRAMEWORK}

As stated in previous sections, this work presents a mathematical programming formulation aiming to determine the optimal production strategy and well layout design for flooding mature fields through polymer injection. A set of surrogate, subsurface models are embedded in the formulation in order to achieve an accurate representation of the reservoir response. These models comprise the $\mathrm{Koval}^{29}$ and capacitance resistance frameworks, ${ }^{36}$ which are explained in more detail in this section. Furthermore, a novel geometric abstraction of the sweep volumes is proposed to make it possible to solve the problem with mathematical programming techniques in reasonable computational times. The geometrical approach is also developed in this section, along with other modeling features.

3.1. Production Forecast Models. Capacitance resistance models (CRM) establish an analogy between the oil reservoir and a network of resistors and capacitors (RC) in an electric circuit to model the connection and response time between every pair of injector and producer wells. While fluid flows are driven by pressure difference, electron flows (current) are caused by potential difference. Additionally, porous media offer 
a resistance to the flow, the same as an electric conductor does on a circuit. Both systems may be also capable of storing mass and energy: oil and displacing fluids may accumulate in the porous media, while the circuit may store electrons on the capacitors. Taking advantage of these analogies, a CRM is capable of predicting the performance of a reservoir. This is a widely used representation with an enormous potential on reservoir simulation and analysis, having shown great results as a general modeling framework. ${ }^{27}$

In few words, the aim is to predict the distribution of the flow injected in an injector well $i$ toward the producers $j$ and the delay in response times through two basic parameters: (a) the connectivity between every pair of wells $\left(f_{i, j}\right)$ and (b) the associated time constant $\left(\tau_{i, j}\right)$. An adapted form of the model is presented in eq 1. Detailed derivation and further theoretical details can be found in the works by Yousef et al., ${ }^{36}$ Dinh and Tiab, $^{39}$ and Kaviani et al. ${ }^{41}$

$$
\begin{aligned}
& \mathrm{QT}_{i, j, t}=\mathrm{QT}_{i, j, t-1} \mathrm{e}^{-\Delta t / \tau_{i, j}}+\left(1-\mathrm{e}^{-\Delta t / \tau_{i, j}}\right) f_{i, j} \mathrm{QI}_{i, t} \\
& \forall i \in I, j \in J, t \in T
\end{aligned}
$$

In eq $1, \mathrm{QT}_{i, j, t}$ is the contribution of injector $i$ to the total production of producer $j$ at time $t, \mathrm{QI}_{i, t}$ refers to the injection rate of injector $i$ at time $t, \Delta t$ is the time length between time points $t-1$ and $t$, and $f_{i, j}$ and $\tau_{i, j}$ are model parameters to be determined by injection-production history matching. Of these parameters, $f_{i, j}$ represents the fraction of the injected fluid in the injector $i$ conducted through the reservoir to the producer $j$, while $\tau_{i, j}$ (the time constant) is related to the dissipation of injection flows in the porous media. More specifically, the time constant refers to the time it takes to achieve $63.2 \%$ of the final production rate at a producer $j$ due to the injection on $i$ (see Figure 3).

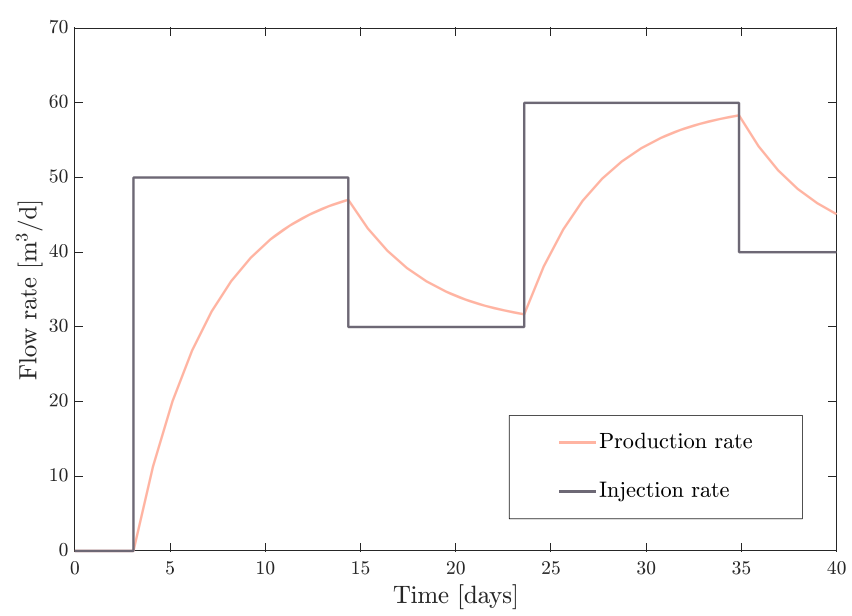

Figure 3. Relationship between injection and production rates with a time constant equal to 4 days modeled by the capacitance resistance model.

Notice that the summation of connectivity factors $f_{i, j}$ over all producers connected to a single injector, despite being a sum of proportions, might be less than unity if reservoir losses exist and could eventually be greater than unity if other production sources are present (e.g., an aquifer). The latter case is not contemplated in this work, and for parametrization, we assume that the summation equals one for every injector. Finally, observe that CRM is a single-phase model, and therefore, a complementary fractional flow model is required in order to assess fluid composition over time, i.e., oil production.

The Koval model ${ }^{29}$ is an extensively used representation that has been mainly developed to predict the core-scale oil productivity from miscible displacement techniques using solvent injection. Specifically, the Koval theory is developed to assess performance deterioration due to fingering effects, a typical issue of many miscible displacement EOR and waterflooding techniques. Fingering effects imply that the displacing fluid bypasses the stored crude oil, leading to early breakthrough of the solvent and poor sweep efficiency. ${ }^{42}$ Physically, fingering effects are driven by rock heterogeneity and differences between the viscosity of the displacing fluid and the crude oil. The Koval model describes fingering effects with a simple factor named $K_{v}$, which is obtained by multiplying the heterogeneity factor $(\mathrm{Hk})$ and the effective mobility ratio between the solvent and the oil $(E)$. Using this characterization, the Koval theory allows making simple estimations of crude oil and solvent proportions in the produced flow as a function of accumulated amounts of the solvent already injected in the reservoir. Typically, larger values of $K_{\mathrm{v}}$ mean that unfavorable heterogeneity and viscosity effects are occurring, and the recovery efficiency is rather poor. A comprehensive physical derivation of the Koval factor using petrophysical data and vertical equilibrium theory ${ }^{43}$ has been presented by Salazar and Lake, ${ }^{34}$ from solid foundations.

The Koval model may be applied to any kind of oil displacing technique under the assumption of a segregated flow between displacing and displaced fluids. ${ }^{28}$ To estimate oil production, $\mathrm{Koval}^{29}$ modifies the viscosity ratio in the fractional-flow equation by Buckley and Leverett ${ }^{43}$ to account for fingering effects. If crude oil and displacing phases show straight-line relative permeabilities, then solvent and oil flows are segregated ${ }^{31}$ and the concentration (or fractional flow) of the solvent can be denoted as shown in eq 2 .

$$
f_{s}=\frac{1}{1+\frac{1}{K_{v}}\left(\frac{1-S}{S}\right)}
$$

where

$$
\begin{aligned}
& K_{\mathrm{v}}=\mathrm{Hk} \cdot E \\
& E=\left[0.78+0.22\left(\frac{\mu_{\mathrm{o}}}{\mu_{\mathrm{s}}}\right)^{1 / 4}\right]^{4} \\
& S=\frac{S_{\mathrm{w}}-S_{\mathrm{wr}}}{1-S_{\mathrm{wr}}-S_{\mathrm{or}}}
\end{aligned}
$$

In these equations, $f_{s}$ refers to the solvent fractional flow (in units of solvent per unit of produced fluid), $S$ is the reduced water saturation defined in eq $5, K_{\mathrm{v}}$ stands for the Koval factor, $E$ computes the effective viscosity ratio, $\mathrm{Hk}$ is a measure of the reservoir heterogeneity, and $\mu_{\mathrm{o}}$ and $\mu_{\mathrm{s}}$ are crude oil and solvent viscosities, respectively. $S_{\mathrm{wr}}$ is the irreducible solvent saturation, and $S_{\text {or }}$ stands for residual oil saturation. Given that polymer flooding techniques usually comprise the injection of an aqueous solution with a relatively low concentration of a polymer, solution saturation may be analogously treated as water saturation. ${ }^{14}$ Water and oil saturation refers to the fraction of water and oil in the effective pore space of the volume under control. This model yields proper predictions on the oil "cut" as a function of the total amount of the solvent 
already injected, with the following inputs: reservoir heterogeneity (from reservoir characterization), initial water saturation, actual water saturation, and effective mobility ratios.

Summarizing, CRM stands as a robust method for quantifying the flows going from injectors to producers, while the Koval theory provides a validated tool to assess solvent and crude oil cuts on the total production flows over time. Both models are plugged into the mathematical programming formulation proposed in this work. Other technical considerations are also taken into account and described in the following sections.

3.2. Geometric Abstraction and Characterization of the Sweep Volumes between the Wells. A novel approach is deployed in this section to efficiently address further technical details of the problem. A geometric-spatial abstraction is introduced for reservoir modeling as well as for tracking polymer slugs. This abstraction consists of the representation of every interwell sweep volume (region between the bottomholes of an injector and producer wells) as a unidirectional pipeline, discretized into regular polyhedrons with homogeneous petrophysical properties. More specifically, we rely on a unidimensional grid discretization, setting a finite number of control points at the center of every block along the flow stream (see Figure 4). Block sizes can be selected according to the

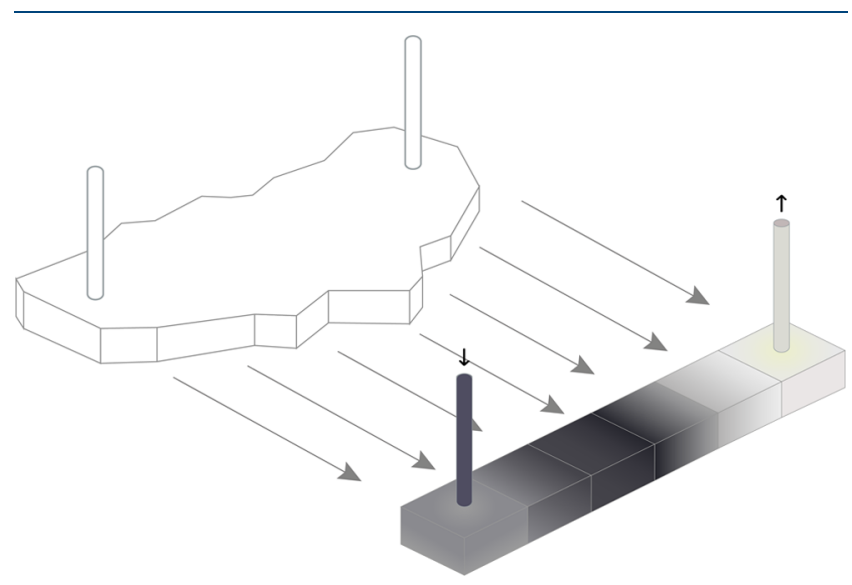

Figure 4. Geometric abstraction of an interwell sweep volume.

technical characterization of the reservoir. Interactions between the sweep volumes of different pairs or links are assumed to be negligible as long as there is sufficient distance between well locations. If this assumption is not totally applicable, then artificial linkages that capture these interactions can be added to the model. This polyhedral-pipeline approach allows discretizing the porous media between wells to gain control over the space and model the behavior of aqueous and oil phases over time.

On the other hand, the time horizon is also discretized accordingly so that the time it takes to move a slug from one block to the next one is exactly one time period. Hence, response times to injection signals are implicitly considered by the model. In other words, for a fixed time discretization, the higher the time constant associated to an injector-producer link, the greater is the number of volumetric blocks in which the sweep volume needs to be divided so that a proper approximation of the associated response time is achieved.

Let $i \in I$ and $j \in J$ denote potential injection and producer wells, respectively, while $j \in J_{i}$ identify production wells connected to injector $i$. Let $u \in U_{i, j}$ stand for discrete volumetric elements in the geometric-spatial abstraction of the sweep region between the bottomholes of wells $i$ and $j$. Let $t \in T$ be the time steps (each of them typically comprising 1 to 10 days) associated to the discretization of the planning horizon. Likewise, let $k \in K$ stand for longer periods (typically months) in which the same planning horizon is discretized so that $t \in$ $\mathrm{TS}_{k}$ denote the short time periods $t$ comprised in the long time period $k$. Note that the number of blocks between two wells and their characteristics are dependent on the interwell distance, the response times, and the heterogeneity of the porous media. Finally, uf $\in U_{i, j}$ denotes the last volumetric block on the edge between $i$ and $j$, directly connected to the production well $j$.

Based on these sets, some parameters are introduced. Let $\mathrm{sw}_{i, j, u, t}^{0}$ stand for the average water saturation at the volumetric block $u$ along the link between wells $i$ and $j$, whereas $s_{\text {or }}$ and $s_{\mathrm{wr}}$ denote residual oil saturation and irreducible water saturation of the reservoir, respectively. Let $\phi_{i, j, u}$ stand for the average porosity of each volumetric block $u$ comprising the link between $i$ and $j$, and $\Delta w_{i, j}, \Delta l_{i, j}$, and $h_{i, j}$ refer to the width, length, and layer thickness of the blocks between $i$ and $j$, respectively. Furthermore, $\mu_{\mathrm{oil}}$ and $\mu_{\mathrm{w}}$ express reservoir oil and pure water viscosity, respectively. It should be noted that many parameters can be indexed such that the model achieves a more accurate representation of each volumetric block linking the wells. Moreover, the model may be extended to a multilayer representation by adding the layer index to each parameter.

Connectivity data are also needed to address this problem. Detailed guidelines on how to obtain connectivities between wells can be found in Yousef et al., ${ }^{36}$ Sayarpour et al., ${ }^{18}$ Moreno and Lake, ${ }^{44}$ Cao et al., ${ }^{28}$ Eshraghi et al., ${ }^{25}$ and Yousefi et al. ${ }^{23}$ Connectivity magnitude is represented by the parameter $\mathrm{cf}_{i, j}$ accounting for the fraction of polymer solution from injector well $i$ that contributes to the production of well $j$. Since the number of wells to operate is not known beforehand, $\mathrm{cf}_{i, j}$ is assumed to be the minimum connectivity when all potential wells operate at the same time. A flow redistribution pattern is defined in the following sections allowing increasing connectivity values according to the number of production wells actually linked to an injector. Figure 5 shows a multiwell representation of the geometric abstraction presented in this section.

\section{OPTIMIZATION MODEL}

4.1. Objective Function. In this section, a mathematical programming model is presented aiming to achieve optimal planning and operation strategies in technical and economic terms. Thus, a performance measure is defined as the net

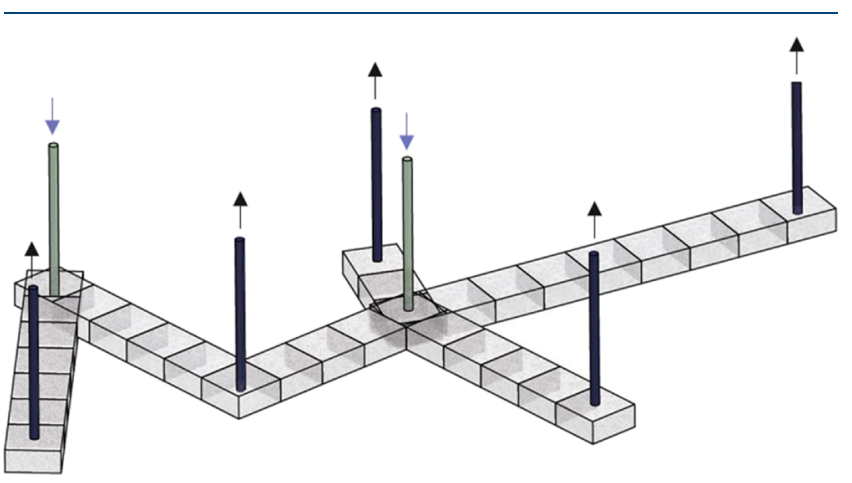

Figure 5. Geometric representation of sweep volumes between two injection wells and five production wells. 
present value of the project considering revenues from oil sales, discounted operational and capital expenditures, and purchase costs of polymers. The objective function is represented by eq 6.

$$
\begin{aligned}
\max z= & \sum_{k \in K}\left\{\frac { 1 } { ( 1 + r ) ^ { k } } \sum _ { t \in T S _ { k } } \left[\operatorname{op}_{k} \mathrm{QTP}_{t}^{\mathrm{oil}} \Delta t\right.\right. \\
& -\sum_{i \in I}\left(\mathrm{pc}_{k} \mathrm{Q}_{i, t}^{\mathrm{IN}} \mathrm{Cp}_{i, t}^{\mathrm{IN}} \Delta t-\operatorname{slcsc}_{i, t}-\text { woi yic }_{i, t}\right) \\
& \left.\left.-\sum_{j \in J}\left(\text { woj yjc }_{j, t}+\operatorname{wcc}_{\mathrm{QAQP}}, \Delta t\right)\right]\right\}
\end{aligned}
$$

In eq $6, \mathrm{op}_{k}$ denotes the oil price $\left(\$ / \mathrm{m}^{3}\right)$ forecasted for time period $k, \mathrm{QTP}_{t}^{\text {oil }}$ refers to the oil production rate $\left(\mathrm{m}^{3} /\right.$ day $)$ during time step $t, \Delta t$ is the duration of time step $t$ (day), $\mathrm{pc}_{k}$ stands for the polymer cost forecasted for time period $k(\$ / \mathrm{kg})$, $Q_{i, t}^{\mathrm{IN}}$ is the injection rate planned for well $i$ at time step $t\left(\mathrm{~m}^{3} /\right.$ day), and $C \mathrm{p}_{i, t}^{\mathrm{IN}}$ indicates the polymer concentration of the slug being injected into well $i$ at time step $t(\mathrm{~g} / \mathrm{L})$. The parameter slc is the slug changeover cost $(\$)$, whereas the slug shift (meaning a change in polymer concentration) in well $i$ at time step $t$ is denoted by the binary variable $\mathrm{sc}_{i, t}$. Parameters woi and woj establish capital expenditures (\$) due to work-over tasks that are required to operate injection and production wells, respectively, while $\mathrm{yic}_{i, t}$ and $\mathrm{yjc}_{j, t}$ are binary variables stating that injector well $i$ or production well $j$ starts operating at time step $t$. Finally, the parameter wcc accounts for the unit processing cost of the produced water $\left(\$ / \mathrm{m}^{3}\right)$, and the continuous variable $\mathrm{QAQP}_{j, t}$ represents the production rate of the aqueous phase $\left(\mathrm{m}^{3} /\right.$ day $)$ in the producer $j$ during time period $t$. The parameter $r$ refers to the interest rate used to evaluate the project profitability.

4.2. Multiphase Transport Equations. In reservoir simulations, polymer flooding transport equations are usually modeled as a set of partial differential equations taking into account three main flow balances through the porous media: the oil phase, water phase, and polymer concentration. While the first two can be described through differential pressure and saturation changes, polymer tracking also adds a diffusion phenomenon driven by the spatial gradient of concentration. Nevertheless, polymer diffusion is expected to cause a minor effect in comparison with pressure difference, particularly when the solvent is pushed at high pressures. Thus, diffusion terms are considered negligible for the approach proposed in this work.

In this context, if the described blocks comprising the sweep volume between every pair of wells are correctly sized, then we may assume that during a single time step $t$, the slug moves ahead one block. Based on this, for each specific volumetric element defined between two wells, a comprehensive mass balance is made, as shown in the following sections.

\subsubsection{Polymer Component Balance.}

$$
\begin{aligned}
& \mathrm{Cp}_{i, j, u, t}^{\text {in }}=\mathrm{Cp}_{i, t}^{\mathrm{IN}} \\
& \forall i \in I, j \in J_{i}, u \in U_{i, j}: u=1, t \in T \\
& \mathrm{Cp}_{i, j, u+1, t+1}^{\text {in }}=\mathrm{Cp}_{i, j, u, t}^{\text {out }} \\
& \quad \forall i \in I, j \in J_{i}, t<|T|, u \in U_{i, j}: u<\left|U_{i, j}\right|
\end{aligned}
$$

$$
\begin{aligned}
& \mathrm{Cp}_{i, j, u, t}^{\text {out }}=\mathrm{Cp}_{i, j, u, t}^{\text {in }}-\mathrm{Crp}_{i, j, u, t} \\
& \forall i \in I, j \in J_{i}, u \in U_{i, j}, t \in T
\end{aligned}
$$

Equation 7 stands for a polymer concentration balance and indicates that for every volumetric block located in the first position on the path between wells $i$ and $j$, the inflow concentration $C \mathrm{p}_{i, j, u, t}^{\text {in }}$ at time step $t$ will be determined by the slug concentration $C \mathrm{p}_{i, t}^{\mathrm{IN}}$ being injected into well $i$ at the same period. In addition, the inflow concentration for any block $u+1$ at period $t+1$ on the sweep volume between $i$ and $j$ is determined by the outflow concentration $\mathrm{Cp}_{i, j, u, t}^{\text {out }}$ of the previous block $u$ at period $t$ (see eq 8 ). The polymer adsorption phenomenon is taken into account in this point. In eq 9, the concentration of the displacing fluid entering block $u$ is reduced to the outflow concentration due to the polymer adsorption phenomenon. Adsorption computation is given by the nonlinear constraint specified in eq 10 , where $a$ and $b$ are specific parameters defined in advance, ${ }^{45}$ along with reservoir characterization, and $\mathrm{Cp}_{i, j, u, t}$ stands for the average polymer concentration over block $u$ on the path $i-j$ at time step $t$. The estimation of $\mathrm{Cp}_{i, j, j, t}$ is addressed in the latter sections.

$$
\begin{aligned}
& \operatorname{Crp}_{i, j, u, t} \geq \frac{a \mathrm{Cp}_{i, j, u, t}}{1+b \mathrm{Cp}_{i, j, u, t}} \\
& \quad \forall i \in I, j \in J_{i}, t \in T, u \in U_{i, j}
\end{aligned}
$$

The polymer retention phenomenon is perceived as highly dependent on the polymer concentration of the solvent being injected and poses a computational challenge since it is driven by a nonconvex constraint.

4.2.2. Displacing Flow Balance. In parallel, the following equations impose material balances for the aqueous phase of the solution being injected.

$$
\begin{gathered}
\mathrm{Qs}_{i, j, u=1, t}^{\mathrm{in}} \leq \mathrm{cf}_{i, j} Q_{i, t}^{\mathrm{IN}}+\sum_{j^{\prime} \in J} \frac{\mathrm{cf}_{i, j^{\prime}}}{\mathrm{nc}_{i}} \mathrm{QU}_{i, j^{\prime}, t}^{\mathrm{IN}} \\
\forall i \in I, j \in J_{i}, t \in T
\end{gathered}
$$

Equation 11 limits the flow rate that enters the first volumetric block on the path between wells $i$ and $j$, at time step $t$. The flow rate along the path $i-j$ is ruled by the connectivity coefficient established for the well pair $i-j$ itself, while a second term distributes the flow predicted for those production wells $j^{\prime}$ that are not operating at time step $t$ (nonnegative variable $\mathrm{QU}_{i, j^{\prime}, t}^{\mathrm{IN}}$ ). Note that $\mathrm{QU}_{i, j, t}^{\mathrm{IN}}$ is defined as an auxiliary variable to address the flow redistribution when a producer $j$ connected to injector $i$ is not operated (shut-in), which is a model decision. From the non-negativity condition of this variable $\left(\mathrm{QU}_{i, j, t}^{\mathrm{IN}} \geq 0\right)$ and as imposed by eq $14, \mathrm{QU}_{i, j, t}^{\mathrm{IN}}$ is enforced to be equal to zero when $\mathrm{yj}_{j, t}$ (binary variable stating that well $j$ is operated during time period $t$ ) is equal to one. On the contrary, if producer $j$ is not operated during time step $t\left(\mathrm{yj}_{j, t}\right.$ $=0)$, then the variable $\mathrm{QU}_{i, j, t}^{\mathrm{IN}}$ takes a positive value, equal to the injection rate in well $i$ during the same time period, as imposed by eqs 12 and 13. The maximum number of production wells that might be linked to the injector $i$ is denoted by the parameter $\mathrm{nc}_{i}$. Notice that if every potential production well $j$ connected to the injection well $i$ operates at time step $t$, the second term on the right side of eq 11 is omitted since all variables $\mathrm{QU}_{i, j^{\prime}, t}^{\mathrm{IN}}$ are equal to zero. Else, for each production well linked to $i$ and being inactive at time step $t$, part 
of the tapped flow is directed equitably to the sweep volumes of active paths.

$$
\begin{aligned}
& \mathrm{QU}_{i, j, t}^{\mathrm{IN}} \geq Q_{i, t}^{\mathrm{IN}}-\mathrm{yj}_{j, t} q_{\mathrm{M}}^{\mathrm{IN}} \quad \forall i \in I, j \in J_{i}, t \in T \\
& \mathrm{QU}_{i, j, t}^{\mathrm{IN}} \leq \mathrm{Q}_{i, t}^{\mathrm{IN}} \quad \forall i \in I, j \in J_{i}, t \in T \\
& \mathrm{QU}_{i, j, t}^{\mathrm{IN}} \leq\left(1-\mathrm{yj}_{j, t}\right) q_{\mathrm{M}}^{\mathrm{IN}} \quad \forall i \in I, j \in J_{i}, t \in T
\end{aligned}
$$

An alternative approach is also possible by distributing the inactive flows to the remaining active wells proportionally to the relative importance of their connectivity coefficients, as shown in eq 15. The latter approach, despite providing a more rigorous representation, has the disadvantage of increasing the computational complexity of the MINLP model.

$$
\begin{aligned}
& \mathrm{Qs}_{i, j, u=1, t}^{\text {in }} \leq \mathrm{cf}_{i, j} Q_{i, t}^{\mathrm{IN}}+\sum_{j^{\prime} \in J} \frac{\mathrm{cf}_{i, j}}{\sum_{j^{\prime \prime} \in J} \mathrm{cf}_{i, j^{\prime \prime}} \mathrm{yj}_{j^{\prime \prime}, t}} \mathrm{cf}_{i, j^{\prime}} \mathrm{QU}_{i, j^{\prime}, t}^{\mathrm{IN}} \\
& \forall i \in I, j \in J_{i}, t \in T
\end{aligned}
$$

Finally, material balances at every block $u$ are completed by eqs $16-18$.

$$
\begin{aligned}
& \mathrm{Qs}_{i, j, u, t}^{\text {out }}=\mathrm{Qs}_{i, j, u, t}^{\text {in }}-Q_{i, j, u, t}^{\text {oil }} \\
& \forall i \in I, j \in J_{i}, t \in T, u \in U_{i, j} \\
& \mathrm{Qs}_{i, j, u+1, t+1}^{\text {in }}=\mathrm{Qs}_{i, j, u, t}^{\text {out }} \\
& \quad \forall i \in I, j \in J_{i}, t<|T|, u \in U_{i, j}: u<\left|U_{i, j}\right| \\
& Q_{j, t}^{\text {OUT }}=\sum_{i: j \in J_{i}}\left(\mathrm{Qs}_{i, j, u f, t}^{\text {out }}+\mathrm{QP}_{i, j, t}^{\text {oil }}\right) \\
& \forall j \in J, u f \in U_{i, j}, t \in T
\end{aligned}
$$

The variable $Q_{i, j, u, t}^{\text {oil }}$ represents the oil volume produced by the slug passing through the block $u$ in the path between wells $i$ and $j$, during time step $t$. The value of this variable is calculated by the Koval model and is described in further sections. $\mathbf{Q s}_{i, j, u, t}^{\text {out }}$ stands for the flow of the solvent leaving block $u$ at time step $t$. Note that a volumetric balance is implicitly stated by eq 16 , driven by the liquid incompressibility assumption. The variable $Q_{i, t}^{\text {OUT }}$ represents the total production of the oil and solvent at well $j$ during time step $t$, including all flows coming from the last volumetric element in every path $i-j$. Note that given that a further part of the flow is now composed of oil, more solvent remains in the reservoir, increasing the water saturation over each block, as shown in the following section.

Water/solution production rates in each producer $j$ during time period $t$ can be obtained from the sum of the aqueous flows leaving the last block uf in every path converging to producer $j$, as shown in eq 19.

$$
\text { QAQP }_{j, t}=\sum_{i: j \in J_{i}} \mathrm{Qs}_{i, j, u f, t}^{\text {out }} \quad \forall j \in J, \text { uf } \in U_{i, j}, t \in T
$$

4.2.3. Physical Conditions in Every Block over Time. Once overall flow balances have been established, quantifying the average polymer concentration within each block is needed to compute several variables, including oil productivity. Considering that retention phenomena (mainly adsorption) could take place with greater intensity in the upstream section of each block, a weighted average between incoming and outgoing conditions is proposed, as shown in eqs 20 and 21 . Note that the concentration of the polymer in the outflow will be lower than that in the incoming flow due to polymer adsorption effects. However, the adsorption is expected to be more pronounced in the first part of the control volume, driven by greater concentration gradients between the flow and the porous media. Typical values for $\alpha_{\mathrm{IN}}$ and $\alpha_{\mathrm{OUT}}$ are 0.55 and 0.45 , respectively.

$$
\begin{gathered}
\mathrm{Cp}_{i, j, u, t}=\alpha_{\mathrm{IN}} \mathrm{Cp}_{i, j, u, t}^{\text {in }}+\alpha_{\mathrm{OUT}} \mathrm{Cp}_{i, j, u, t}^{\text {out }} \\
\forall i \in I, j \in J_{i}, t \in T, u \in U_{i, j} \\
\alpha_{\mathrm{IN}}+\alpha_{\text {OUT }}=1 ; \alpha_{\text {OUT }}>\alpha_{\mathrm{IN}}
\end{gathered}
$$

Finally, the viscosity of the displacing fluid is computed through eq 22. To achieve an accurate value of this critical variable, a cubic polynomic equation ${ }^{46}$ is used, according to the polymer concentration. Although different salt concentrations can be considered by using the Flory-Huggins ${ }^{46}$ correlation, we assume a constant salinity of the water being used.

$$
\begin{gathered}
\mu_{i, j, u, t}^{\mathrm{P}}=\mu^{\mathrm{W}}\left(1+\gamma_{1} \mathrm{Cp}_{i, j, u, t}+\gamma_{2} \mathrm{Cp}_{i, j, u, t}^{2}+\gamma_{3} \mathrm{Cp}_{i, j, u, t}^{3}\right) \\
\forall i \in I, j \in J_{i}, t \in T, u \in U_{i, j}
\end{gathered}
$$

In the latter equation, $\mu_{i, j, u, t}^{\mathrm{P}}$ is the average solvent viscosity over the block $u$ at period $t$, while $\mu^{\mathrm{W}}$ refers to pure water viscosity. $\gamma_{1}, \gamma_{2}$, and $\gamma_{3}$ are the specific coefficients that better fit the viscosity-concentration curve, depending on the type of polymer and the salinity of the water being used. Displacing fluid viscosity stands as a critical variable due to the main premise of the polymer flooding process aimed at improving the mobility ratio by adjusting viscosity and keeping physical phenomena under control.

4.2.4. Oil Productivity from Every Block. As explained in Section 3.2, the Koval theory is used to predict the oil recovery by combining two essential parameters: one denoting the viscosity contrast between oil and water phases and the other accounting for the heterogeneity of the reservoir. Both of them are used to build the Koval factor, which allows making accurate predictions on the amount of oil being recovered. The heterogeneity factor is assumed to be given from the flow capacity and storage capacity analysis of the reservoir and is denoted by $\mathrm{Hk}$ in the formulation. This parameter can also be estimated from permeability and porosity data, as proposed by Salazar and Lake. ${ }^{34}$

On the other hand, viscosity contrast between displaced and displacing fluids is considered through the effective mobility ratio, denoted in this work by Ev. This parameter is usually defined as a function of the viscosity ratio of the fluids, as shown in eq $23 .^{29}$

$$
\begin{gathered}
\operatorname{Ev}_{i, j, u, t} \geq\left[0.78+0.22 \sqrt[4]{\frac{\mu^{\mathrm{OIL}}}{\mu_{i, j, u, t}^{\mathrm{P}}}}\right]^{4} \\
\forall i \in I, j \in J_{i}, t \in T, u \in U_{i, j}
\end{gathered}
$$

Note that in terms of the optimization problem, $\operatorname{Ev}_{i, j, u, t}$ constitutes a variable being computed for every volumetric block $u$ in the sweep volume between $i$ and $j$ at each time period $t$, given its dependence on the viscosity of the displacing fluid 
(slug). The parameter $\mu^{\mathrm{OIL}}$ refers to the viscosity of the crude oil in the reservoir.

The effective mobility ratio and the heterogeneity factor are both inputs for the Koval factor estimation, as presented in eq 24. Finally, the flow composition (usually referred to as the fractional flow) is given in terms of eqs 25 and 26, from the Koval theory. ${ }^{29}$

$$
\begin{gathered}
\mathrm{Kv}_{i, j, u, t}=\mathrm{Ev}_{i, j, u, t} \mathrm{Hk} \quad \forall i \in I, j \in J_{i}, t \in T, u \in U_{i, j} \\
\mathrm{Fs}_{i, j, u, t} \geq \frac{1}{1+\frac{1}{\mathrm{Kv}_{i, j, u, t}}\left(\frac{1-\mathrm{rSw}_{i, j, u, t}}{\mathrm{rSw}_{i, j, u, t}}\right)} \\
\forall i \in I, j \in J_{i}, t \in T, u \in U_{i, j} \\
\mathrm{rSw}_{i, j, u, t}=\frac{\mathrm{Sw}_{i, j, u, t}-s_{\mathrm{wr}}}{1-s_{\mathrm{wr}}-s_{\mathrm{or}}} \\
\forall i \in I, j \in J_{i,}, t \in T, u \in U_{i, j}
\end{gathered}
$$

In eq $25, \mathrm{Fs}_{i, j, u, t}$ represents the fraction of the displacing fluid (aqueous polymer solution) in the total flow across the block $u$ of path $i-j$ during period $t$, while $\mathrm{rSw}_{i, j, u, t}$ is a continuous variable ranging between 0 and 1 and defined according to eq 26 as the reduced water saturation of each block over time. Recall that $s_{\mathrm{wr}}$ and $s_{\mathrm{or}}$ stand for irreducible water saturation and residual oil saturation parameters of the reservoir, respectively. Finally, $\mathrm{Sw}_{i, j, u, t}$ refers to the water saturation on each volumetric block $u$ between $i$ and $j$ during time step $t$. Indeed, the latter variable needs to be updated period by period. To achieve that, water saturation is initialized from reservoir characterization and then computed by eqs 27 and 28 .

$$
\begin{gathered}
\mathrm{Sw}_{i, j, u, t=1}=\mathrm{sw}_{i, j, u}^{0} \quad \forall i \in I, j \in J_{i}, t \in T, u \in U_{i, j} \\
\mathrm{Sw}_{i, j, u, t+1}=\mathrm{Sw}_{i, j, u, t}+\frac{\left(\mathrm{Qs}_{i, j, u, t}^{\mathrm{IN}}-\mathrm{Qs}_{i, j, u, t}^{\mathrm{OUT}}\right) \Delta t}{\phi_{i, j, u}^{\mathrm{e}} v_{i, j}^{\mathrm{GB}}} \\
\forall i \in I, j \in J_{i}, t \in T, u \in U_{i, j}
\end{gathered}
$$

where

$$
v_{i, j}^{\mathrm{GB}}=\Delta w_{i, j} \Delta l_{i, j} h_{i, j} \quad \forall i \in I, j \in J_{i}
$$

In these equations, $v_{i, j}^{\mathrm{GB}}$ is the volume of each individual block defined between $i$ and $j\left(\mathrm{~m}^{3}\right)$, and $\phi_{i, j, u}^{\mathrm{e}}$ stands for the effective porosity that can be estimated as in eq 30 , where ipv refers to the average inaccessible pore volume proportion and $\phi_{i, j, u}$ is the total porosity.

$$
\begin{aligned}
\phi_{i, j, u}^{\mathrm{e}}= & (1-\mathrm{ipv}) \phi_{i, j, u} \\
& \forall i \in I, j \in J_{i}, u \in U_{i, j}
\end{aligned}
$$

Once again, based on the liquid incompressibility assumption, as the flow progresses across successive blocks extracting oil, an equivalent volume of the solvent remains in the block, increasing water saturation and reducing oil saturation. More specifically, eq 28 updates the water saturation variable by adding the ratio of the equivalent volume of oil extracted during period $t$ and the free volume within the block. Notice that depending on the amount of the solvent being injected, water saturation growth rates may vary significantly, having negative effects on the oil productivity if effective mobility is not being controlled properly.

4.2.5. Permeability Reduction. Injectivity loss due to permeability reduction effects is also addressed in this model, albeit in a simplified manner. Permeability reduction can be addressed as a function of one of its main causes, that is, the accumulation of the adsorbed polymer. Thus, the accumulated amount of the polymer retained in each volumetric block is computed as shown in eq 31, while for the calculation of the reduction in permeability, a hyperbolic function is proposed, as in eq $32 .^{14}$

$$
\begin{aligned}
& \operatorname{QARP}_{i, j, u, t}=\sum_{t^{\prime}<t} \operatorname{Crp}_{i, j, u, t^{\prime}} \\
& \forall i \in I, j \in J_{i}, t \in T, u \in U_{i, j} \\
& \mathrm{Rk}_{i, j, u, t} \geq 1+\frac{\left(\mathrm{rk}^{\mathrm{MAX}}-1\right) \beta_{\mathrm{Rk}} \mathrm{QARP}_{i, j, u, t}}{1+\beta_{\mathrm{Rk}} \mathrm{QARP}_{i, j, u, t}} \\
& \forall i \in I, j \in J_{i}, t \in T, u \in U_{i, j}
\end{aligned}
$$

In the latter equations, $\operatorname{QARP}_{i, j, u, t}$ stands for the accumulated amount of the polymer per unit volume retained in the block $u$ of path $i-j$ up to time $t ; \mathrm{Rk}_{i, j, u, t}$ quantifies the relative permeability reduction; $\mathrm{rk}^{\mathrm{MAX}}$ refers to the maximum possible value for $\mathrm{Rk}_{i, j, u, t}$. In turn, $\beta_{\mathrm{Rk}}$ is a predefined reservoir engineering parameter standing for the permeability reduction rate for the given polymer being used. ${ }^{47,48}$

Accounting for injectivity issues is highly relevant due to the consequences that they might bring, such as improper propagation of the solvent, borehole enlargement, and/or undesirable fractures that may lead to the leakage of polymers, among other effects. Since injectivity is defined as the injection rate divided by the injection pressure, if injectivity decreases, then either a lower flow is imposed or a higher pressure is required. Thereby, this model takes injectivity into account by lowering the upper bound on the injection rate for injector $i$ at period $t$ according to the permeability reduction in every block of the path, as shown in eq 33 . We note that in general, the first block in the path is the most restricting one.

$$
Q_{i, t}^{\mathrm{IN}} \leq \frac{q_{\mathrm{M}}^{\mathrm{IN}}}{\mathrm{cf}_{i, j} \mathrm{Rk}_{i, j, u, t}} \quad \forall i \in I, j \in J_{i}, t \in T, u \in U_{i, j}
$$

Constraint 33 represents another fact that discourages massive injection of polymers in high concentrations to avoid future issues in the oil recovery driven by the loss of injectivity.

4.2.6. Total Production of Oil over Time. Oil production must be quantified in order to compute the economic income in the objective function (eq 6). Since the fraction of the displacing fluid is defined by eq 25 , the amount of oil produced from each volumetric block can be readily calculated as in eq 34 . In addition, an auxiliary variable $A Q_{i, j, u, t}^{\text {oil }}$ is introduced in eqs 35-37 to track the accumulated amount of oil being recovered by a slug, block by block.

$$
\begin{aligned}
& Q_{i, j, u, t}^{\text {oil }} \leq\left(1-\mathrm{Fs}_{i, j, u, t}\right) \mathrm{Qs}_{i, j, u, t}^{\mathrm{IN}} \\
& \quad \forall i \in I, j \in J_{i}, t \in T, u \in U_{i, j} \\
& \mathrm{AQ}_{i, j, u=1, t}^{\text {oil }}=Q_{i, j, u=1, t}^{\text {oil }} \quad \forall i \in I, j \in J_{i}, t \in T
\end{aligned}
$$




$$
\begin{aligned}
& \mathrm{AQ}_{i, j, u, t}^{\text {oil }}=Q_{i, j, u, t}^{\text {oil }}+\mathrm{AQ}_{i, j, u-1, t-1}^{\text {oil }} \\
& \quad \forall i \in I, j \in J_{i}, u \in U_{i, j}: u>1, t \in T \\
& \mathrm{QP}_{i, j, t}^{\text {oil }}=\mathrm{AQ}_{i, j, u f, t}^{\text {oil }} \quad \forall i \in I, j \in J_{i}, u f \in U_{i, j}, t \in T
\end{aligned}
$$

$$
\mathrm{QTP}_{t}^{\mathrm{oil}} \leq \sum_{i} \sum_{j \in J_{i}} \mathrm{QP}_{i, j, t}^{\mathrm{oil}} \quad \forall t \in T
$$

In eq $38, \mathrm{QP}_{i, j, t}^{\text {oil }}$ stands for the oil production from the path $i-j$ during time step $t$, while $\mathrm{QTP}_{t}^{\text {oil }}$ accounts for total field production during time period $t$. This structure allows oil accumulation along every path in a recursive way, to finally compute the total production of oil that impacts on the objective function.

A set of critical trade-offs to solve should be observed and commented up to this point. First, it must be noticed that viscosity plays a fundamental role in the polymer flooding optimization because the effective mobility ratio directly impacts on the composition of the production flow. To favor effective mobility ratios (and, as a result, the oil fraction in the outflow), viscosity must be set as close as possible to crude oil viscosity. A cubic polynomial approach has been proposed in order to compute viscosity against different polymer concentrations in the injected solution. The coefficients of the polynomial must be defined in advance and will vary according to the salinity of the water and the molecular weight and type of polymer being used (see Figure 6). In fact, for a viscous-oil

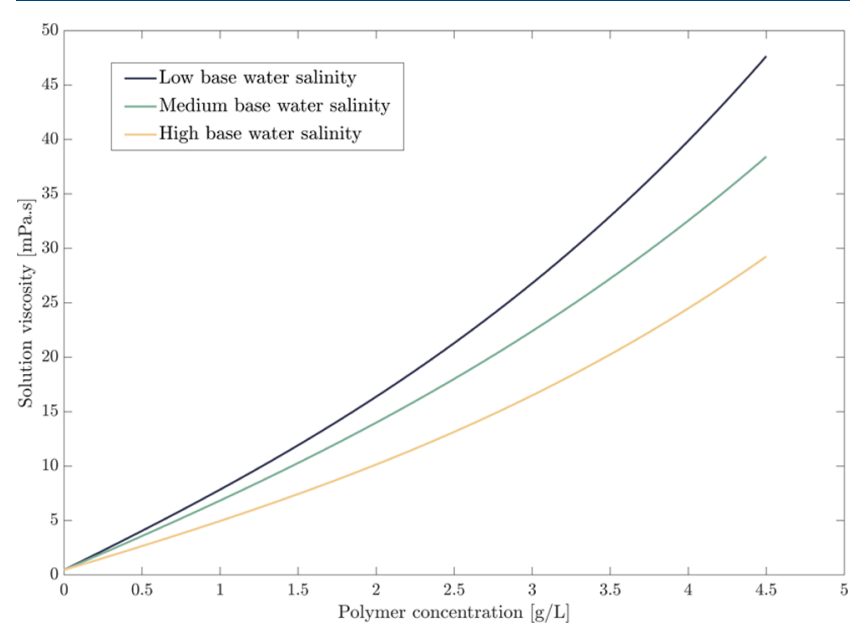

Figure 6. Viscosity behavior with respect to polymer concentration at different base water salinities. Salinity affects the polymer structure and reduces its capacity to form cross-links. Adapted from ref 49 . Copyright 2019 Multidisciplinary Digital Publishing Institute.

reservoir, base viscosity (water) may need to be multiplied 100 times to reach trapped crude oil viscosity. To achieve this, higher polymer concentrations are needed, with the reciprocal increase in costs, adsorption phenomena, reduction of permeability, and injectivity issues.

Although higher viscosities are desirable to improve mobility ratios and, hence, sweep efficiency, polymer adsorption needs also to be controlled. At higher polymer concentrations, polymer adsorption phenomena are increased, and as polymer retention increases, permeability is decreased (Figure 7) also reducing injectivity. This changes the original rates and pressures at which fluids can be pumped into the reservoir

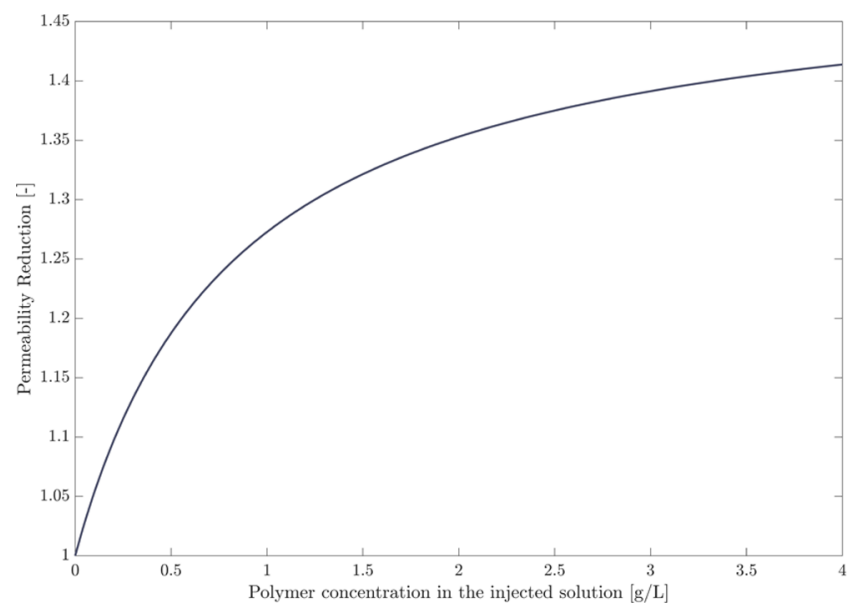

Figure 7. Permeability reduction as a function of the polymer concentration in the solution being injected.

without fracturing the formation. Reservoir characterization, rock properties, and bottomhole pressure (BHP) studies will determine the parametrization of $a, b$, and $\beta_{\mathrm{Rk}}$ in eqs 10 and 32 . Depending on this parametrization, permeability reduction might result in a drop of the injection rate of up to $40 \%$. These effects might hardly condition greedy strategies, to ensure the safety of the formation.

At the same time, dependence of the oil productivity on water saturation is also critical. In this regard, it must be noticed that the higher the recovery rate over time, the faster the water saturation is increased within the reservoir, reducing the extraction capacity in more encouraging periods of peak oil prices or higher demand. Fractional flow dependence on reduced water saturation and the effective mobility ratio is shown in Figure 8. Notice that the blue area stands for the desirable operating area, with relevant amounts of oil being extracted.

4.3. Well Selection and Slug Change. As seen in eqs 12 to 15 , binary variables are required in order to account for the wells actually being operated at a certain time step $t$. Nevertheless, additional equations to identify the time at which each well starts operating are also needed to compute expenditures associated with work-over tasks (if the well already exists) or drilling and completion tasks (if the well is new) at the right time. To model this, eqs 39 to 42 are added to the formulation. Equations 39 and 40 identify the time at which an injection or production well starts operating, by assigning the value one to a binary variable (yic or yjc) when the operation variable (yi or yj) changes from 0 to 1 . In turn, eqs 41 and 42 do not allow shutting down an operating well during the planning horizon after it starts working. We plan to relax the latter assumption in future work.

$$
\begin{aligned}
& \text { yic }_{i, t}=\mathrm{yi}_{i, t}-\mathrm{yi}_{i, t-1} \quad \forall i \in I, t \in T \\
& y_{j, t}=y j_{j, t}-y_{j, t-1} \quad \forall j \in J, t \in T \\
& \mathrm{yi}_{i, t} \geq \mathrm{yi}_{i, t-1} \quad \forall i \in I, t \in T \\
& \mathrm{yj}_{j, t} \geq \mathrm{yj}_{j, t-1} \quad \forall j \in J, t \in T
\end{aligned}
$$

Additionally, continuous variables such as the injection rate, the injected fluid concentration, and production rates at every 


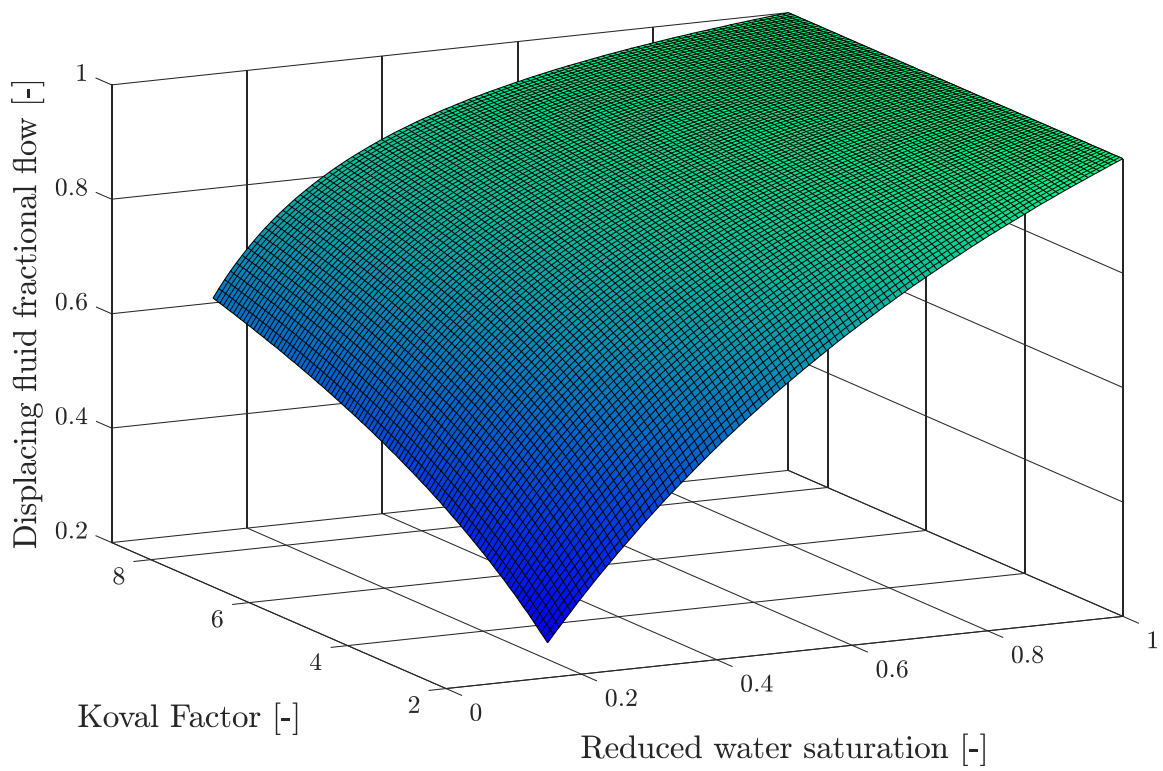

Figure 8. Fractional flow of the displacing fluid as a function of the Koval factor and the reduced water saturation.

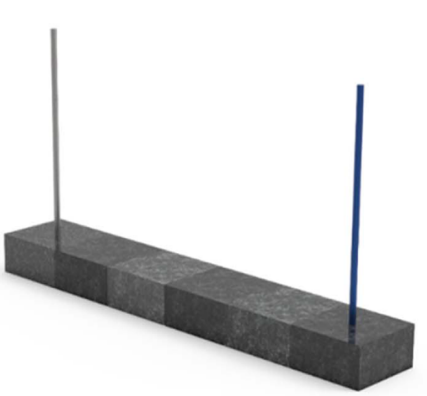

(a)

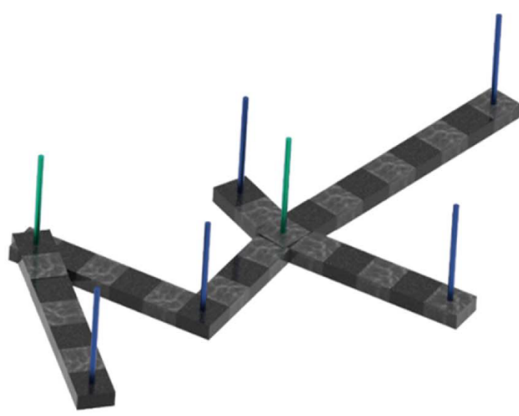

(b)

Figure 9. (a) Six discretized blocks connecting two wells for Case Study 1. (b) Two injection and five production wells connected by six paths, divided into a total of thirty six blocks (Case Study 2 ).

well must be subject to its operation status, as imposed by eqs 43 to 46 .

$$
\begin{aligned}
& Q_{i, t}^{\mathrm{IN}} \leq q_{\mathrm{M}}^{\mathrm{IN}_{i, t}} \quad \forall i \in I, t \in T \\
& \mathrm{Q}_{j, t}^{\mathrm{OUT}} \leq q_{\mathrm{M}}^{\mathrm{OUT}} \mathrm{yj}_{j, t} \quad \forall j \in J, t \in T \\
& \mathrm{Cp}_{i, t}^{\mathrm{IN}} \leq \mathrm{cp}^{\mathrm{MAX}_{\mathrm{yi}}} \quad \forall i \in I, t \in T \\
& \mathrm{Qs}_{i, t, t, t}^{\mathrm{IN}} \leq q_{\mathrm{M}}^{\mathrm{IN}} \mathrm{yj}_{j, t} \quad \mathrm{Qs}_{i, j, u, t}^{\mathrm{IN}} \leq q_{\mathrm{M}}^{\mathrm{IN}} \mathrm{yi}_{i, t} \\
& \forall i \in I, j \in J_{i}, t \in T, u \in U_{i, j}
\end{aligned}
$$

In eq $45, \mathrm{cp}^{\mathrm{MAX}}$ stands for the maximum concentration of the polymer that may be used in the injected solution.

Finally, additional constraints are stated for slug change computation. Slugs are changed every time the polymer concentration is varied for a specific injection well. Thus, binary variables are required in order to establish changeover timing and costs, as well as slug minimum lengths (see eqs 47 to 53). Binary variables $s 1_{i, t}$ and $s 2_{i, t}$ become strictly equal to 1 when a slug of a higher or lower concentration is selected for injector $i$ at time $t$ (with regard to the previous time step), respectively. $s 3_{i, t}$ is an auxiliary variable stating that the slug remains unchanged. The variable $\mathrm{sc}_{i, t}$ indicates the slug changeover occurrence to be estimated in the objective function, while the parameter $\varepsilon_{\mathrm{Cp}}$ stands for a small tolerance within which the concentration is not considered to be changing.

$$
\begin{aligned}
& \mathrm{Cp}_{i, t+1}^{\mathrm{IN}}-\mathrm{Cp}_{i, t}^{\mathrm{IN}}=\mathrm{SLK}_{i, t}-\mathrm{SLK}_{i, t} \quad \forall i \in I, t \in T \\
& \mathrm{SLK}_{i, t} \geq \varepsilon_{\mathrm{Cp}} \mathrm{s}_{i, t} \quad \forall i \in I, t \in T \\
& \mathrm{SLK}_{i, t} \leq \mathrm{cp}^{\mathrm{MAX}} \mathrm{s}_{i, t} \quad \forall i \in I, t \in T \\
& \mathrm{SLK}_{i, t} \geq \varepsilon_{\mathrm{Cp}} \mathrm{s}_{i, t} \quad \forall i \in I, t \in T \\
& \mathrm{SLK}_{i, t} \leq \mathrm{cp}^{\mathrm{MAX}} \mathrm{s}_{i, t} \quad \forall i \in I, t \in T \\
& \mathrm{sc}_{i, t} \geq \mathrm{s}_{i, t}+\mathrm{s}_{i, t} \quad \forall i \in I, t \in T \\
& \mathrm{~s}_{i, t}+\mathrm{s} 2_{i, t}+\mathrm{s}_{i, t}=1 \quad \forall i \in I, t \in T
\end{aligned}
$$

Note that the duration of a slug is also determined by these binary variables. In fact, the time difference between consecutive $\mathrm{sc}_{i, t}$ that are equal to 1 measures the slug length and might be used for the definition of more constrained strategies. 


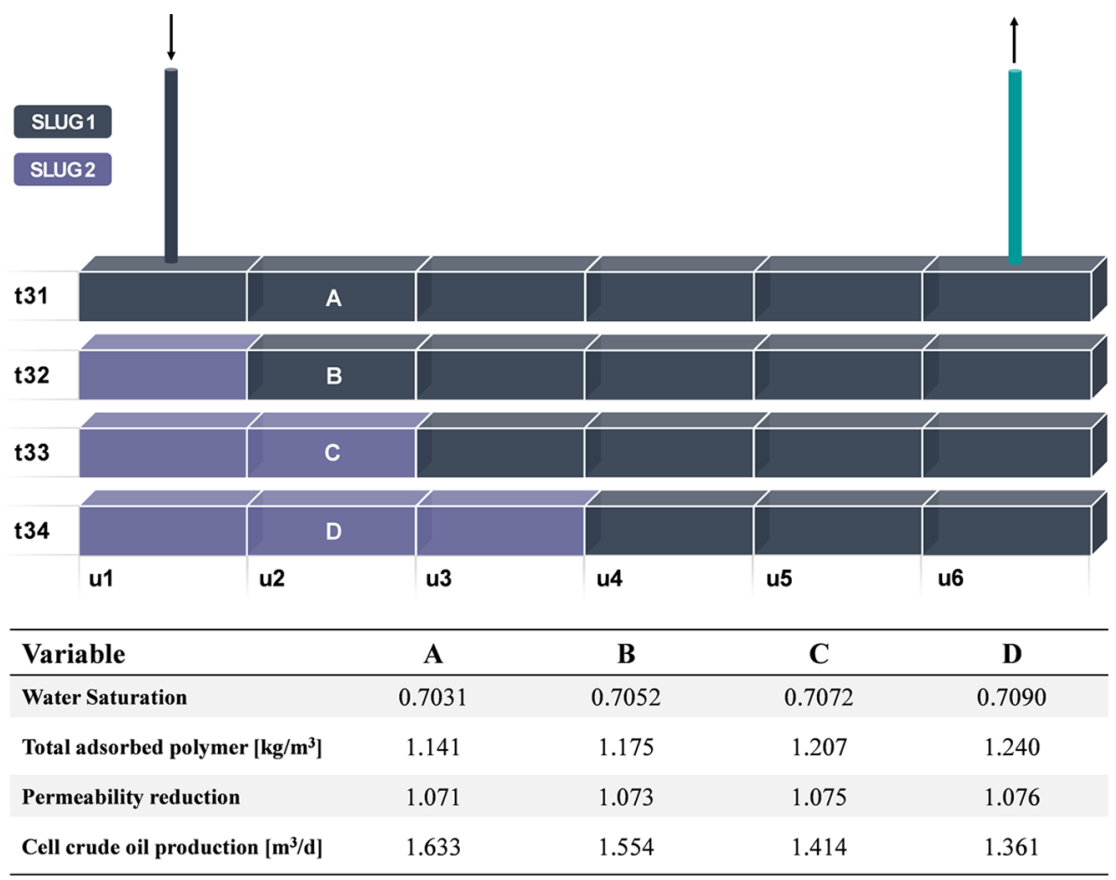

Figure 10. Schematic of the sweep volume between two wells, illustrating the values of state variables after different slugs move through block u2.

Table 1. Computational Details of the Optimization Procedure for Case Studies 1 and 2

\begin{tabular}{|c|c|c|c|c|c|c|}
\hline \multirow[b]{2}{*}{ illustrative case } & \multicolumn{3}{|c|}{ model size } & \multicolumn{3}{|c|}{ subproblem execution } \\
\hline & discrete variables & continuous variables & number of equations & subproblem type & CPU time (s) & major iterations \\
\hline \multirow[t]{2}{*}{ Case Study 1} & 447 & 9403 & 10,079 & NLP & 31 & 4 \\
\hline & & & & MILP & 28 & 3 \\
\hline \multirow[t]{2}{*}{ Case Study 2} & 1980 & 70,618 & 73,398 & NLP & 1255 & 5 \\
\hline & & & & MILP & 13,114 & 4 \\
\hline
\end{tabular}

\section{RESULTS}

Two illustrative cases are presented in this section to test the model and validate the results. The first study case consists of a single injector well and a single producer well connected through a sweep volume divided into six discrete elements. The second case presents a set of two possible injection wells and five potential producers comprising an irregular geometric arrangement (see Figure 9). It should be noted that the arrays of blocks are obtained from actual drain volumes for each pair of injection-production wells. The procedure to discretize the volumetric domain is as follows: we consider the projected sweep volumes between the bottomholes of every pair of potential injection and production wells. For each pair of wells, we estimate the response time to injection signals. We then adopt a fixed time period length (in our case, 4 days) and determine the number of blocks between the wells assuming that the slugs move one block per time step. Notice that if a finer discretization is required for a certain pair of wells, then the timescale needs to be modified accordingly. Connectivity and time constants are usually derived from a history-matching procedure, according to CRM premises. ${ }^{27,36,50}$ Finally, for each block, average petrophysical properties must be estimated, such as initial water saturation, effective porosity, irreducible water and residual oil saturation, and reservoir heterogeneity characterization according to the Koval theory. ${ }^{29}$ This information from reservoir engineering is usually available after waterflooding. In both cases, we assume that the displacing fluid is an aqueous solution of hydrolyzed polyacrylamide (HPAM) with an effective salinity of 1 equiv per liter. ${ }^{51}$

5.1. Case Study 1. In this section, a simple case study consisting of two wells operating as injection and production wells is considered. Well operation (yes/no) decisions are dismissed in this illustrative case. The effective sweep volume between the wells is modeled by 6 volumetric blocks of 12,500 $\mathrm{m}^{3}$ each. The distance between the wells is about $300 \mathrm{~m}$, and response time of the production well to an injection signal is estimated to be 24 days. Details on specific technical parametrization for this example are presented in Tables S2 and S3 of the Supporting Information. Notice that according to the mathematical formulation presented in Section 4, whenever a slug passes through a volumetric block, water saturation, reduced water saturation, polymer adsorption, and permeability conditions are updated for the next time step. These state variables determine the sweeping potential of the next time step and, therefore, oil recovery efficiency. Technical decision variables such as the injection rate and polymer concentration will define the viscosity of the injected fluid and the effective mobility ratio, which allows computing the oil production in every block according to the Koval theory. Figure 10 illustrates the updating of state variables, showing the evolution of the second block (u2) in the path between two wells, from time steps $\mathrm{t} 31$ to $\mathrm{t} 34$.

The planning horizon for this case study is set at 1 year, divided into 90 periods of 4 days each. For illustration, fixed oil and polymer prices of $400 \$ / \mathrm{m}^{3}$ and $4 \$ / \mathrm{kg}$ are considered over 


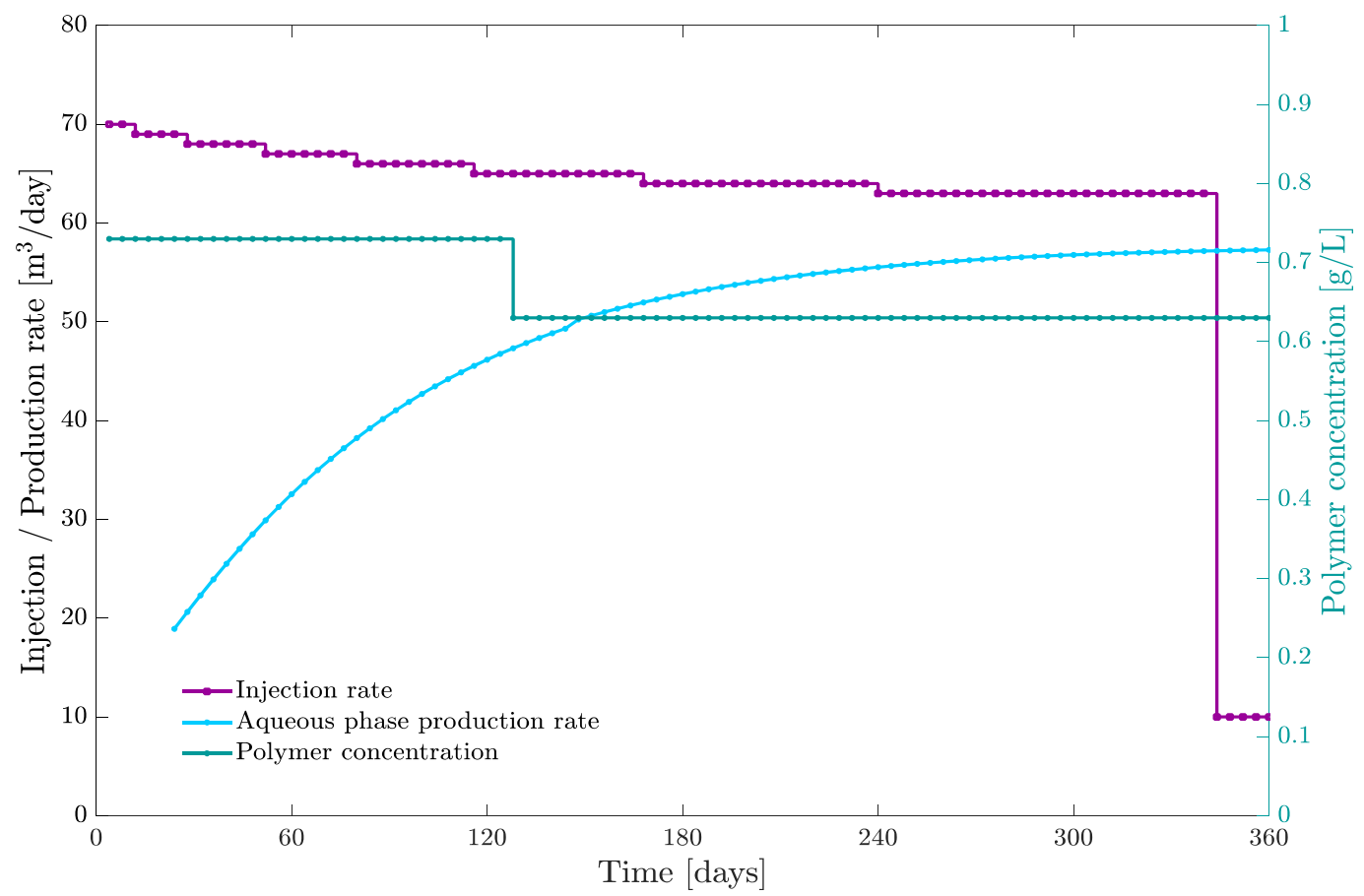

Figure 11. Polymer concentration in the injected solution, injection rate, and aqueous phase production rate over time, resulting from the best found solution to Case Study 1.
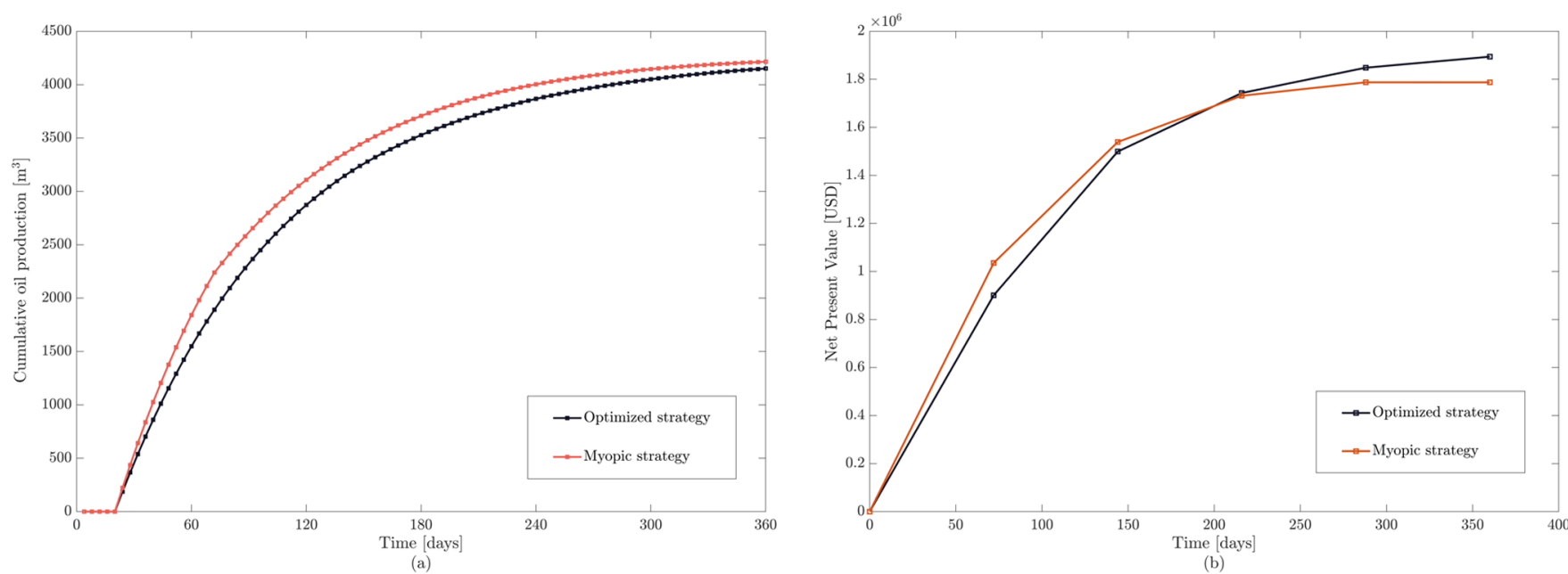

Figure 12. (a) Cumulative oil production driven by the optimized EOR strategy vs the myopic strategy. (b) Cumulative net present value for both strategies over time.

the whole planning horizon in order to avoid speculative effects in the production strategy. This assumption filters out solutions that might tend to accelerate production on peak periods to take advantage of oil prices. Nevertheless, the model is capable of managing variations in the oil price over time.

The model is coded in the software GAMS v31.1 ${ }^{52}$ and solved with the solver DICOPT. DICOPT stands for an MINLP optimization algorithm based on outer approximation, equality relaxation, and augmented penalty strategies. ${ }^{53}$ Since the DICOPT algorithm succesively solves NLP and MIP subproblems, we use CONOPT to solve NLP subproblems and CPLEX to address MIP master problems. Due to the nonconvex nature of several constraints in the model, the optimizer does not necessarily obtain the global optimum. Elapsed time for the solution of Case Study 1 is around $59 \mathrm{~s}$ on a Windows PC with an Intel Core i7 3.6 GHz, 8-core processor and 16 GB RAM. Additional details on the model size and computational performance are presented in Table 1 .

The results of this case study show that the best found strategy implies the use of two slugs during the planning horizon (see Figure 11). The injection rate is initially set at its maximum value and gradually decreases for safety reasons driven by the loss of injectivity. While the first slug has a duration of approximately 120 days, the second slug, of lower polymer concentration, lasts until the end of the time horizon. Note that in order to keep high injection rates that allow a greater recovery of crude oil over the whole time horizon, the concentration must be limited to prevent damaging the well injectivity.

The model is also used to forecast oil production and assess the net present value of the EOR project when compared to a myopic strategy, injecting polymers at a high rate, using a 
Table 2. Interwell Connectivity (CF) and the Number of Volumetric Blocks (NVB) between Wells for Case Study 2

\begin{tabular}{|c|c|c|c|c|c|c|c|c|c|c|}
\hline \multirow{2}{*}{$\frac{\text { producer }}{\text { injector }}$} & \multicolumn{2}{|c|}{$\mathrm{J} 1$} & \multicolumn{2}{|c|}{$\mathrm{J} 2$} & \multicolumn{2}{|c|}{$\mathrm{J} 3$} & \multicolumn{2}{|c|}{$\mathrm{J} 4$} & \multicolumn{2}{|c|}{ J5 } \\
\hline & CF & NVB & CF & NVB & CF & NVB & CF & NVB & CF & NVB \\
\hline I1 & 0.7 & 7 & 0.3 & 6 & 0 & 0 & 0 & 0 & 0 & 0 \\
\hline I2 & 0 & 0 & 0.2 & 5 & 0.3 & 6 & 0.1 & 3 & 0.4 & 9 \\
\hline
\end{tabular}

reasonably high concentration of $2.5 \mathrm{~g} / \mathrm{L}\left(\mathrm{g}\right.$ per $\mathrm{L}$ or $\mathrm{kg}$ per $\mathrm{m}^{3}$ ) over the whole time horizon. As observed in Figure 12, the myopic strategy, usually seen in practice, might produce a slightly higher accumulated volume of crude oil at the end of the time horizon. More specifically, while the myopic production strategy delivers a cumulative output of $4208 \mathrm{~m}^{3}$ of oil, the optimized strategy yields a cumulative production of $4160 \mathrm{~m}^{3}$ after one year ( $1 \%$ less). Nevertheless, the net present value of the cash flows at the end of the planning horizon shows an additional benefit of $\$ 1.12 \times 10^{5}$ in favor of the optimized strategy $(6.2 \%$ more $)$. While the optimized strategy yields a net present value of $\$ 1.895 \times 10^{6}$, the myopic strategy throughput stays at $\$ 1.783 \times 10^{6}$.

Despite the fact that nonconvex constraints in the model might lead to solutions that are not globally optimal, it is worth mentioning that a mixed-integer linear programming (MILP) relaxation of the original model was deployed and tested for Case Study 1. The MILP model considers a wider feasible region from the outer approximation of nonlinear functions with piecewise linear counterparts. More specifically, ad hoc piecewise linear approximations with a maximum of 3 linear segments have been used to relax nonlinear constraints. Results show that the optimized solution from the MINLP is just 5\% worse than the relaxed (infeasible) solution yielded by the MILP model in terms of the NPV. To summarize, it is possible to state that the optimized solution obtained for Case Study 1 is at most 5\% below the global optimal solution. Moreover, if a better solution exists, then the relevance of optimized injection strategies with respect to myopic strategies may be even larger than reported.

5.2. Case Study 2. Case study 2 involves 36 volumetric blocks linking two potential injection wells and five potential producers. Well selection and operation decisions become relevant in this case. The effective sweep volume between wells is modeled as shown in Figure 9, using a different number of blocks depending on the dimensions of the sweep volume along each path and the corresponding response time. Similar to Case Study 1 , all blocks are set at a volume of $12,500 \mathrm{~m}^{3}$. The spatial layout, the number of blocks, and the connectivity between injection and production wells for the flow distribution are given in Table 2 and Figure 13. Other technical parameters used in this case study, as well as illustrative maps of initial water saturation, permeability, and pressures, are presented in Tables S4 and S5 and Figures S2-S4 of the Supporting

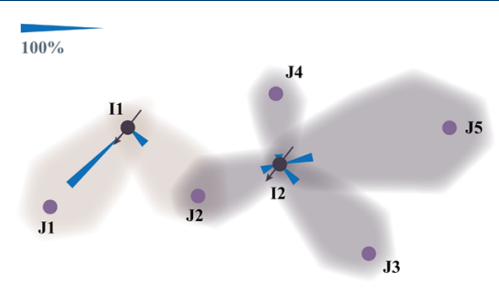

Figure 13. Potential well layout and connectivity representation for Case Study 2. Colored regions represent projected drain areas around the wells.
Information. The initial polymer concentration in each volumetric block is assumed to be zero. Fixed oil prices and polymer cost are set for the entire planning horizon.

The optimization model for this case study is also coded in the software GAMS 31.1 and solved with DICOPT using CONOPT for NLP subproblems and CPLEX for MIP master problems. Solving the model to an optimality gap of 0.01 takes around 14,369 s (4 h) using CONOPT on the same PC referred in the previous section. Additional details on the model size and computational performance are also presented in Table 1.

Optimized injection rates and polymer concentration of the injected solution over time are presented in Figure 14. It should be noted that the injector I1 operates with three different slugs during the time horizon, while injector $\mathrm{I} 2$ operates most of the time using the same polymer concentration. In terms of production wells, all but $\mathrm{J} 4$ are suggested for operation by the optimized results. Furthermore, every selected well operates from the beginning of the project.

Given that the maximum allowable injection rate is the same as for Case Study 1 but the injected flow is distributed through different paths to two or more production wells, higher polymer concentrations can be injected without significantly affecting the well injectivity. In this way, a greater number of connections allow the adsorption phenomenon not to be concentrated in a certain path. Injectivity restrictions are evidenced in the injection plan for well I1, on which the injection rate is reduced after 160 days to increase the polymer concentration during the second half of the year. The so-called end-of-horizon effect is observed in the final periods of the planning horizon, where polymer concentration is reduced to avoid economic expenditures that do not report an economic return before the completion of the planning horizon. Although this issue can be mitigated by implementing longer planning horizons, computational costs may increase significantly. Alternatively, providing ending conditions for the decision variables or implementing a rolling horizon framework ${ }^{54}$ can effectively address the effect.

With regard to the economic value of the optimized strategy, the forecasted production rate of crude oil for each producing well is presented on Figure 15. In addition, because the producer $\mathrm{J} 2$ shares incoming flows from two injection wells, Figure 15 also shows the production breakdown due to each injector. The oil production rate over the field during the planning horizon is presented in Figure 16, as well as its cumulative value over time. Cumulative oil production for the optimized strategy is estimated at $21,920 \mathrm{~m}^{3}$ with a net present value of $\$ 6.68 \times 10^{6}$.

It is important to note that well selection decisions may have a huge impact on the project's economic results and the overall recovery of oil. Taking advantage of the most promising sweeping regions to the detriment of those that are not justified can make a big difference in redistributing the flows and sweeping off the richer areas with a higher flow rate. For clarity, we have also made a comparison of the optimized strategy with the myopic strategy that uses a constant polymer concentration of $2.5 \mathrm{~g} / \mathrm{L}$, at the maximum injection rate, operating all available 

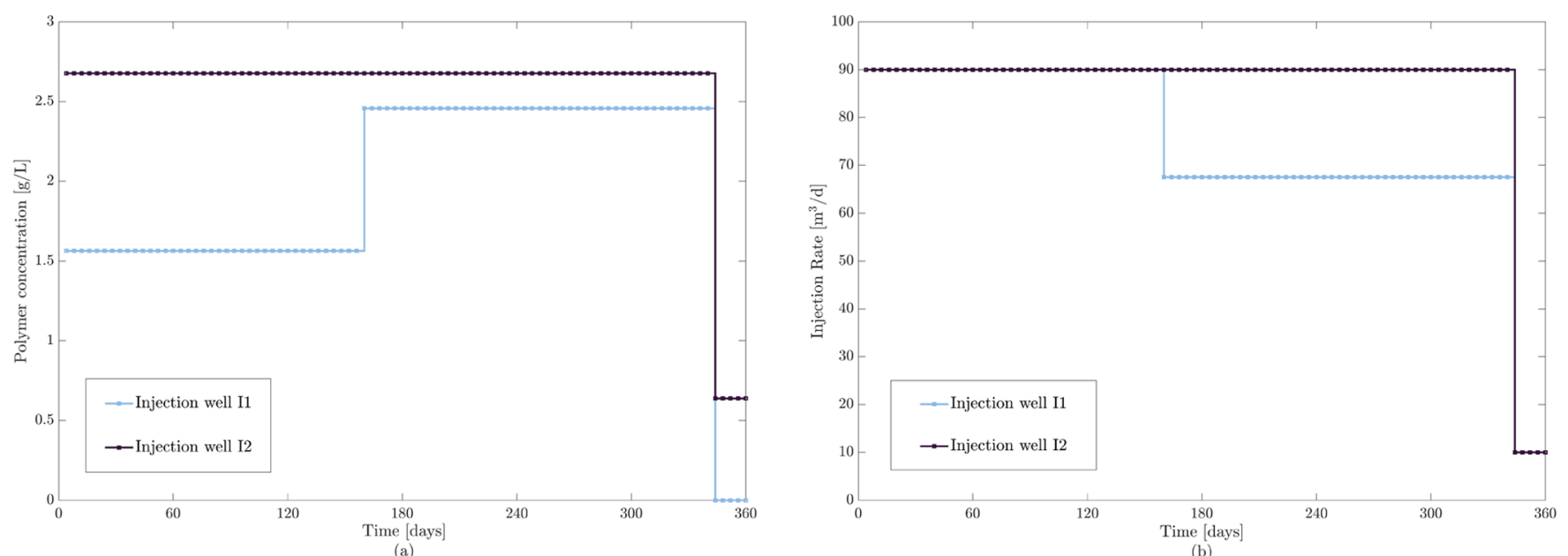

Figure 14. (a) Polymer concentration of the injected solution for each injection well. (b) Optimal injection rate for each injection well.
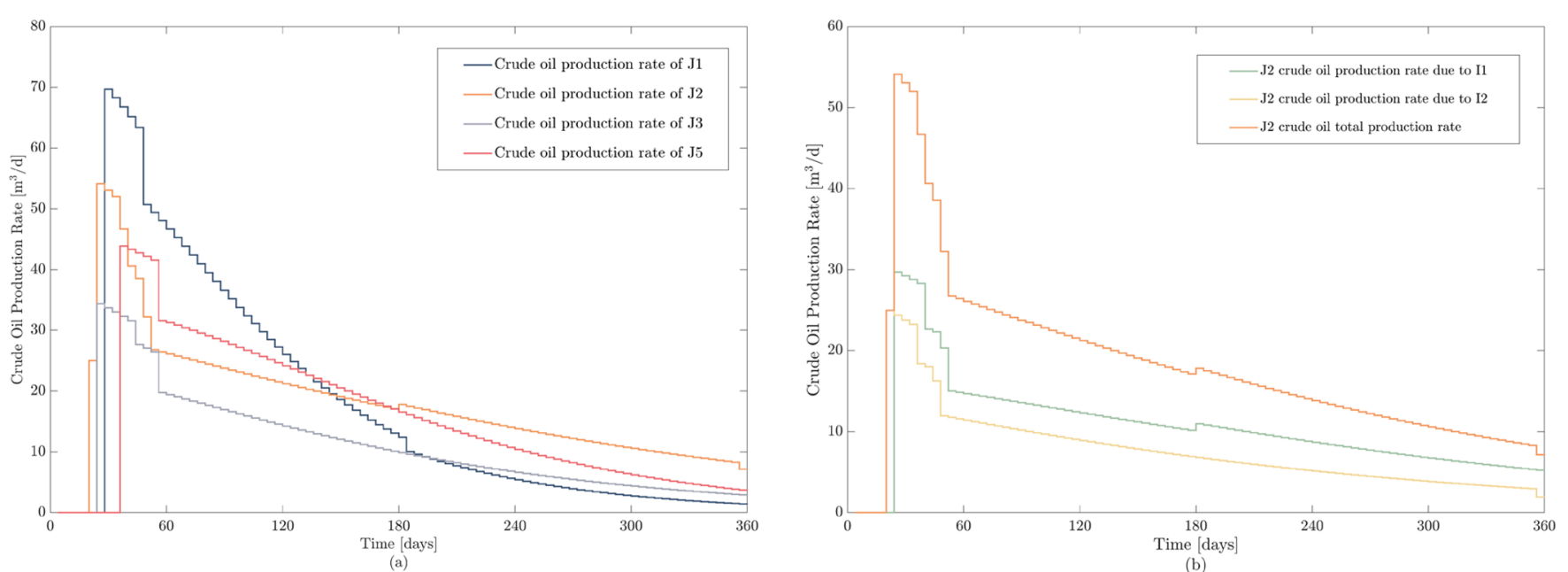

Figure 15. (a) Crude oil production rate for each producing well forecasted by the optimization model following the optimized injection strategy. (b) Production from well J2: oil rate breakdown according to the injection well.

wells from the beginning of the time horizon. Unlike Case Study 1 , cumulative oil production of the optimized strategy is slightly superior to the myopic strategy, reaching a $2 \%$ higher ultimate recovery. Economically, however, the difference is far greater (see Figure 17). By avoiding the investment in the well $\mathrm{J} 4$ and achieving a faster recovery, the optimized strategy yields a net present value of $\$ 6.68 \times 10^{6}$ against $\$ 5.71 \times 10^{6}$ of the myopic strategy ( $17 \%$ more). Scaling the problem to larger fields can provide even more value by optimizing well selection strategies and injection plans.

\section{CONCLUSIONS}

A decision-making tool based on MINLP mathematical programming has been developed in order to face critical aspects of the oil field design and production planning for enhanced oil recovery through polymer flooding. The model is meant to serve as a technical and economical evaluation tool during the screening phase of mature fields, after waterflooding. A novel modeling framework has been proposed, combining existing technical models of proven accuracy with a geometric abstraction for every sweep volume between the wells, in order to reduce the solving complexity. The presented optimization framework can also provide high scalability, allowing incorporation of additional wells and multilayer schemes, the precise mapping of petrophysical properties through finer discretization, the computation of more variables on each volumetric block, and many other features.

In terms of optimization results, the case studies show the relevance of the model to determine convenient production strategies and oil field designs. The additional profit yielded by optimized solutions proves to be significant compared to those yielded by myopic strategies, frequently used in practice. An outstanding sensitivity of the net present value to surface design decisions, such as well selection and operation, has been demonstrated in the experiments conducted in this work. The latter aspect confirms the importance of the field design problem $^{55}$ and leverages the proposed tool as a valuable aid for reservoir engineers. A substantial improvement of $17 \%$ in the net present value is achieved by applying the optimization tool to a set of 7 total wells. It is clear that for fields of a larger scale, well selection decisions become more and more important. On the other hand, optimizing polymer concentration and injection rates provides a safe and efficient way to control many other phenomena, such as polymer adsorption, permeability reduction, and injectivity issues, among others. The modular properties of the optimization framework facilitate the integration of further analytical models to address additional phenomena. 


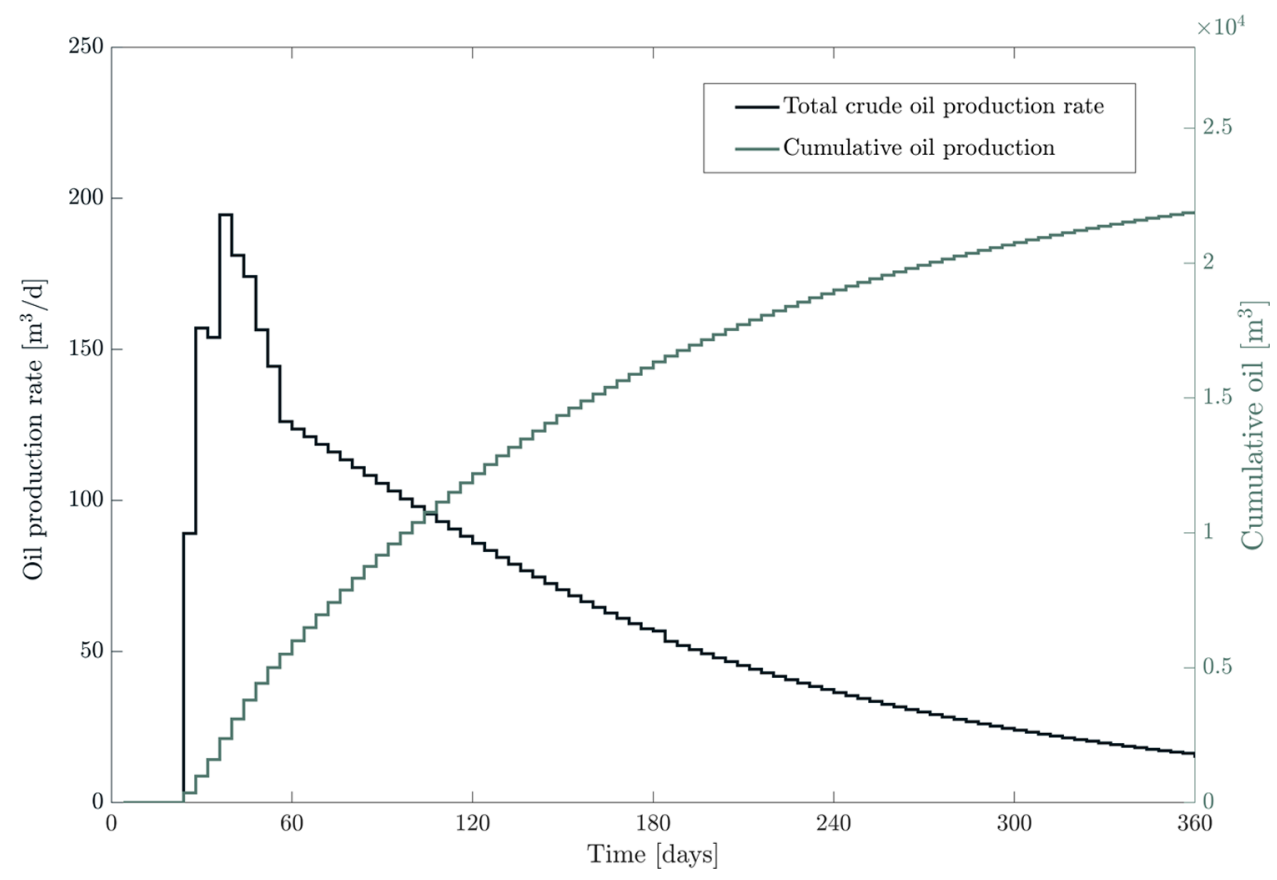

Figure 16. Oil production rate over the field during the planning horizon.

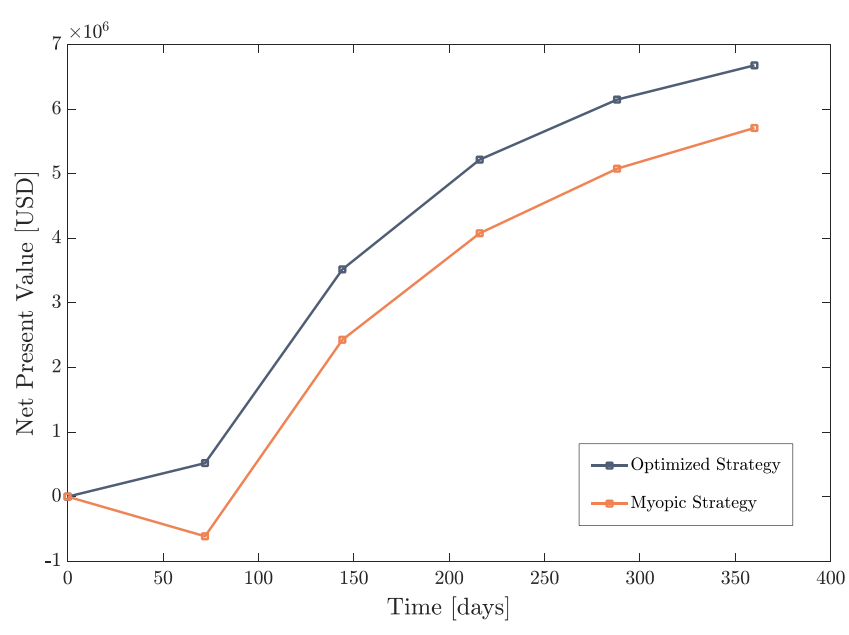

Figure 17. Comparison of the net present value of the optimized strategy (with 6 out of 7 wells being operated) with the myopic strategy where every available well is being operated.

Finally, it is important to mention that the modeling framework may also serve as a forecasting tool for other EOR strategies, for parameter validation in reservoir modeling, and/ or for simple comparison between different operation strategies. The modular structure allows expanding its potential to other EOR techniques, such as $\mathrm{CO}_{2}$ injection, a stimulation strategy favored by carbon capture and sequestration initiatives. To deepen into methodological aspects of the modeling environment, problem resolution procedures and the deployment of statistical tools that allow a greater accuracy in the geometric abstraction of the sweep volumes are also planned as future work.

\section{ASSOCIATED CONTENT}

\section{(s) Supporting Information}

The Supporting Information is available free of charge at https://pubs.acs.org/doi/10.1021/acs.iecr.1c00803.
Table comparing the proposed framework against other optimization approaches; tables presenting the values of the input parameters for both case studies; figures illustrating the noninteracting assumption and estimated reservoir properties for Case Study 2 (PDF)

\section{AUTHOR INFORMATION}

\section{Corresponding Author}

Diego C. Cafaro - INTEC (UNL-CONICET), 3000 Santa Fe, Argentina; Facultad de Ingeniería Química, Universidad Nacional del Litoral, 3000 Santa Fe, Argentina; 10 orcid.org/ 0000-0002-3893-5106; Email: dcafaro@fiq.unl.edu.ar

\section{Authors}

Demian J. Presser - INTEC (UNL-CONICET), 3000 Santa Fe, Argentina; Facultad de Ingeniería Química, Universidad Nacional del Litoral, 3000 Santa Fe, Argentina

Vanina G. Cafaro - INTEC (UNL-CONICET), 3000 Santa Fe, Argentina; Facultad de Ingeniería Química, Universidad Nacional del Litoral, 3000 Santa Fe, Argentina

Complete contact information is available at:

https://pubs.acs.org/10.1021/acs.iecr.1c00803

\section{Notes}

The authors declare no competing financial interest.

\section{ACKNOWLEDGMENTS}

The authors acknowledge financial support from the CONICET under Grant PIP-112 20150641 and from the Universidad Nacional del Litoral under Grant CAI+D 201650420150100101LI. Financial support from the YPF Tecnología S.A. (YTEC) is also gratefully acknowledged.

\section{NOMENCLATURE}

Sets

$i \in I \quad$ potential injection wells

$j \in J \quad$ potential production wells 
$j \in J_{i} \quad$ potential production wells with no-null connectivity with potential injection well $i$

$k \in K$

$t \in T$ longer time periods in the discretized horizon

$T$ time periods in the discretized planning horizon

$t \in \mathrm{TS}(k)$ time periods in the long time period $k$

$u \in U_{i, j} \quad$ discrete volumetric elements between wells

uf $\in U_{i, j} \quad$ last volumetric block along the path between $i$ and $j$

\section{Parameters}

$a, b \quad$ polymer adsorption phenomenon parameters

$\mathrm{cf}_{i, j} \quad$ connectivity between injector well $i$ and producer $j$

$\mathrm{cp}^{\mathrm{MAX}}$

[-]

$\left.\mathrm{cp}^{\mathrm{m}} \quad \mathrm{m}^{3}\right]$

$h_{i, j} \quad$ layer thickness of the volumetric blocks $U_{i, j}[\mathrm{~m}]$

$\mathrm{Hk}$ heterogeneity factor [-]

ipv inaccessible pore volume $[-]$

$\mathrm{nc}_{i} \quad$ maximum number of production wells that might be linked to injection well $i$ during operations

$\mathrm{op}_{k} \quad$ oil price forecasted for time period $k\left[\$ / \mathrm{m}^{3}\right]$

$\mathrm{pc}_{k} \quad$ polymer cost forecasted for time period $k[\$ / \mathrm{kg}]$

$q_{\mathrm{M}}^{\mathrm{IN}} \quad$ upper bound for the injection rate $\left[\mathrm{m}^{3} / \mathrm{day}\right]$

$q_{\mathrm{M}}^{\mathrm{OUT}} \quad$ upper bound for the production rate $\left[\mathrm{m}^{3} /\right.$ day $]$

$r \quad k$-period interest rate [-]

$\mathrm{rk}^{\mathrm{MAX}}$ maximum value for the permeability reduction coefficient [-]

$s_{\text {or }} \quad$ residual oil saturation [-]

slc slug changeover cost [\$]

$\mathrm{sw}_{i, j, u, t}^{0} \quad$ initial water saturation at volumetric block $u$ in the link between $i$ and $j$ during $t[-]$

$s_{\mathrm{wr}} \quad$ irreducible water saturation [-]

$v_{i, j}^{\mathrm{GB}} \quad$ volume of each individual block $u$ in the path $i-j$ $\left[\mathrm{m}^{3}\right]$

wcc unit processing cost for the produced water phase $\left[\$ / \mathrm{m}^{3}\right]$

woi capital expenditures due to work-over tasks to operate an injection well $[\$]$

woj capital expenditures due to work-over tasks to operate a production well $[\$]$

$\alpha_{\mathrm{IN}}, \alpha_{\text {OUT }}$ parameters to compute average polymer concentration within each block $u$

$\beta_{\mathrm{Rk}} \quad$ experimental parameter to compute the permeability reduction coefficient

$\gamma_{1}, \gamma_{2}, \gamma_{3}$ experimental parameters to compute average polymer solution viscosity within each block $u$

$\Delta l_{i, j} \quad$ length of the volumetric blocks $U_{i, j}[\mathrm{~m}]$

$\Delta t \quad$ time length between time points $t-1$ and $t$ [day]

$\Delta w_{i, j} \quad$ width of the volumetric blocks $U_{i, j}[\mathrm{~m}]$

$\varepsilon_{\mathrm{Cp}} \quad$ threshold to determine slug changing $[\mathrm{g} / \mathrm{L}]$

$\mu^{\mathrm{OIL}} \quad$ oil viscosity $[\mathrm{mPa} \mathrm{s}]$

$\mu^{\mathrm{W}} \quad$ pure water viscosity [mPa s]

$\phi_{i, j, u} \quad$ average porosity of each volumetric block $u$ in the link between $i$ and $j[-]$

$\phi_{i, j, u}^{\mathrm{e}} \quad$ effective porosity estimation for each block $u$ in the path between $i$ and $j[-]$

\section{Positive Variables}

$\mathrm{AQ}_{i, j, u, t}^{\text {oil }} \quad$ auxiliary variable to track the accumulated amount of oil being produced on each block $u$ across the link between $i$ and $j$ up to time period $t\left[\mathrm{~m}^{3} /\right.$ day $]$

$C \mathrm{p}_{i, j, u, t}$ average polymer concentration for volumetric block $u$ in the link between $i$ and $j$ during $t[\mathrm{~g} / \mathrm{L}]$

$\mathrm{Cp}_{i, t}^{\mathrm{IN}} \quad$ polymer concentration of the slug being injected into well $i$ at time step $t[\mathrm{~g} / \mathrm{L}]$
$\mathrm{Cp}_{i, j, u, t}^{\text {in }}$

inflow polymer concentration for volumetric block $u$ in the link between $i$ and $j$ during $t[\mathrm{~g} / \mathrm{L}]$

$\mathrm{Cp}_{i, j, u, t}^{\text {out }} \quad$ outflow polymer concentration for volumetric block $u$ in the link between $i$ and $j$ during $t[\mathrm{~g} / \mathrm{L}]$

$\mathrm{Crp}_{i, j, u, t}$ amount of the polymer per unit volume retained in the block $u$ of path $i-j$ during $t\left[\mathrm{~kg} / \mathrm{m}^{3}\right]$

$\mathrm{Ev}_{i, j, u, t} \quad$ effective mobility ratio within the block $u$ in the path between $i$ and $j$ during $t[-]$

$\mathrm{Fs}_{i, j, u, t} \quad$ fraction of the displacing fluid in the total flow across the block $u$ in the path between $i$ and $j$ during $t[-]$

$\mathrm{Kv}_{i, j, u, t} \quad$ Koval factor for the block $u$ in the path between $i$ and $j$ during $t[-]$

$\mathrm{QAQP}_{j, t} \quad$ water phase production rate of producer $j$ during time step $t\left[\mathrm{~m}^{3} /\right.$ day $]$

$\operatorname{QARP}_{i, j, u, t}$ accumulated amount of the polymer per unit volume retained in the block $u$ of the path $i-j$ up to time step $t\left[\mathrm{~kg} / \mathrm{m}^{3}\right]$

$Q_{i, t}^{\mathrm{IN}} \quad$ injection rate at well $i$ during $t\left[\mathrm{~m}^{3} /\right.$ day $]$

$Q_{j, t}^{\text {OUT }}$

$Q_{i, j, u, t}^{\text {oil }}$ production rate at well $i$ during $t\left[\mathrm{~m}^{3} /\right.$ day $]$ oil production rate at the block $u$ in the path between $i$ and $j$ during $t\left[\mathrm{~m}^{3} /\right.$ day $]$

$\mathrm{QP}_{i, j, t}^{\text {oil }}$ oil production of the path $i-j$ during time step $t$ $\left[\mathrm{m}^{3} /\right.$ day $]$

$\mathrm{Qs}_{i, j, u, t}^{\text {in }} \quad$ inflow polymer solution rate for volumetric block $u$ in the link between $i$ and $j$ during $t\left[\mathrm{~m}^{3} /\right.$ day $]$

$\mathrm{Qs}_{i, j, u, t}^{\text {out }}$

$\mathrm{QTP}_{t}^{\text {oil }}$

$\mathrm{QU}_{i, j, t}^{\mathrm{IN}}$

$\mathrm{rSw}_{i, j, u, t}$

$\mathrm{Rk}_{i, j, u, t}$

$\mathrm{Sw}_{i, j, u, t}$ outflow polymer solution rate for volumetric block $u$ in the link between $i$ and $j$ during $t\left[\mathrm{~m}^{3} /\right.$ day] oil production rate of the entire field during time step $t\left[\mathrm{~m}^{3} /\right.$ day $]$ auxiliary continuous variable for the flow distribution $\left[\mathrm{m}^{3} /\right.$ day $]$

reduced water saturation at each block $u$ in the path between $i$ and $j$ during $t[-]$

permeability reduction coefficient $[-]$

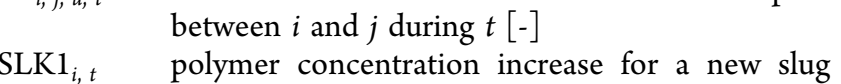
polymer concentration increase
injected in well $i$ at period $t[\mathrm{~g} / \mathrm{L}]$

$\mathrm{SLK} 2_{i, t} \quad$ polymer concentration decrease for a new slug injected in well $i$ at period $t[\mathrm{~g} / \mathrm{L}]$

$\mu_{i, j, u, t}^{\mathrm{P}} \quad$ average displacing fluid viscosity over the block $u$ in the path between $i$ and $j$ during $t[\mathrm{mPa}$ ]

\section{Binary variables}

sc $_{i, t} \quad$ tells the slug shift for injector $i$ at time $t$

$s 1_{i, t} \quad$ indicates a slug shift to a higher concentration for injector $i$ at time step $t$

$s 2_{i, t} \quad$ indicates a slug shift to a lower concentration for injector $i$ at time step $t$

$s 3_{i, t} \quad$ auxiliary variable telling that the slug remains the same for injector $i$ at time step $t$

yic $_{i, t}$ indicates if injection well $i$ starts operating at time step $t$

yjc $_{j, t}$ indicates if production well $j$ starts operating at time step $t$

$\mathrm{yi}_{i, t}$ indicates if injection well $i$ is operating at time step $t$

$\mathrm{yj}_{j, t}$ indicates if production well $j$ is operating at time step $t$

\section{Capacitance Resistance Model}

$f_{i, j} \quad$ connectivity between injector well $i$ and producer $j$

$\mathrm{QT}_{i, j, t}$ production rate of producer $j$ contributed by injector $i$ at time $t\left[\mathrm{~m}^{3} /\right.$ day $]$

$\mathrm{QI}_{i, t}$ injection rate of injector $i$ at time $t\left[\mathrm{~m}^{3} /\right.$ day $]$

$\Delta t \quad$ time length between time points $t-1$ and $t[\mathrm{~d}]$ 
$\tau_{i, j} \quad$ time constant associated with injector $i$ and producer $j$ [d]

\section{Koval Model}

$E$ effective viscosity ratio [-]

$f_{\mathrm{s}}$ solvent fractional flow [-]

$\mathrm{Hk}$ heterogeneity factor [-]

$K_{\mathrm{v}}$ Koval factor [-]

$S$ reduced water saturation $[-]$

$S_{\text {or }}$ residual oil saturation [-]

$S_{\mathrm{wr}}$ irreducible water saturation [-]

$S_{\mathrm{w}}$ water saturation [-]

$\mu_{\mathrm{o}}$ oil viscosity [mPa $]$

$\mu_{\mathrm{s}}$ displacing fluid viscosity $[\mathrm{mPa} \mathrm{s}]$

\section{REFERENCES}

(1) IEA World Energy Outlook 2020, 2020. https://www.iea.org/ reports/world-energy-outlook-2020

(2) Littmann, W. Polymer Flooding; ELSEVIER, 1988.

(3) Lake, L.W. Enhanced Oil Recovery; Prentice Hall, 1989.

(4) Sorbie, K. S. Polymer-Improved Oil Recovery; Springer Publishing: 2013.

(5) de Melo, M. A.; Holleben, C. R.; Silva, I. G.; de Barros Correia, A.; Silva, G. A.; Rosa, A. J.; Lins, A. G.; de Lima, J. C. Evaluation of Polymer-Injection Projects in Brazil. SPE Latin American and Caribbean Petroleum Engineering Conference; Soc. Pet. Eng.: 2005.

(6) Saleh, L. D.; Wei, M.; Zhang, Y.; Bai, B. Data Analysis for Polymer Flooding That Is Based on a Comprehensive Database. SPE Reservoir Eval. Eng. 2017, 20, 0876-0893.

(7) AlSofi, A. M.; Blunt, M. J. Polymer flooding design and optimization under economic uncertainty. J. Pet. Sci. Eng. 2014, 124, $46-59$.

(8) Shafiee, M.; Animah, I.; Alkali, B.; Baglee, D. Decision support methods and applications in the upstream oil and gas sector. J. Pet. Sci. Eng. 2019, 173, 1173-1186.

(9) Wang, D.; Seright, R. S.; Shao, Z.; Wang, J. Key Aspects of Project Design for Polymer Flooding. SPE Annual Technical Conference and Exhibition. 2007.

(10) Horowitz, B.; do Nascimento Guimarães, L. J.; Dantas, V.; Afonso, S. M. B. A concurrent efficient global optimization algorithm applied to polymer injection strategies. J. Pet. Sci. Eng. 2010, 71, 195204.

(11) Williams, C. K. I. Prediction with Gaussian Processes: From Linear Regression to Linear Prediction and Beyond. Learning in Graphical Models; Springer: Dordrecht, 1998, 599-621, DOI: $10.1007 / 978-94-011-5014-923$.

(12) Li, S.; Lei, Y.; Zhang, X.; Zhang, Q. Optimal control solving of polymer flooding based on a hybrid genetic algorithm. Proceedings of the 29th Chinese Control Conference; IEEE: 2010, 5194-5198.

(13) Lei, Y.; Li, S.; Zhang, Q. Optimal control solving of polymer flooding based on real-coded genetic algorithm. 2010 8th World Congress on Intelligent Control and Automation; IEEE: 2010, 51115114.

(14) Lei, Y.; Li, S.; Zhang, X.; Zhang, Q.; Guo, L. Optimal control of polymer flooding based on mixed-integer iterative dynamic programming. Int. J. Control 2011, 84, 1903-1914.

(15) Ekkawong, P.; Han, J.; Olalotiti-Lawal, F.; Datta-Gupta, A. Multiobjective design and optimization of polymer flood performance. J. Pet. Sci. Eng. 2017, 153, 47-58.

(16) Janiga, D.; Czarnota, R.; Stopa, J.; Wojnarowski, P.; Kosowski, P. Performance of nature inspired optimization algorithms for polymer Enhanced Oil Recovery process. J. Pet. Sci. Eng. 2017, 154, 354-366.

(17) ECLIPSE Reservoir Simulation Software; Reference Manual. Schlumberger: 2014, Retrieved from https://www.software.slb.com/ products/eclipse.

(18) Sayarpour, M.; Kabir, C. S.; Lake, L. W. Field Applications of Capacitance-Resistance Models in Waterfloods. SPE Reservoir Eval. Eng. 2009, 12, 853-864.
(19) Lee, K. H.; Ortega, A.; Nejad, A. M.; Ershaghi, I. An Active Method for Characterization of Flow Units Between Injection/ Production Wells by Injection-Rate Design. SPE Reservoir Eval. Eng. 2011, 14, 433-445.

(20) Parekh, B.; Kabir, C. S. A case study of improved understanding of reservoir connectivity in an evolving waterflood with surveillance data. J. Pet. Sci. Eng. 2013, 102, 1-9.

(21) Tafti, T. A.; Ershaghi, I.; Rezapour, A.; Ortega, A. Injection Scheduling Design for Reduced Order Waterflood Modeling. SPE Western Regional \& AAPG Pacific Section Meeting 2013 Joint Technical Conference; Soc. Pet. Eng.: 2013.

(22) Mamghaderi, A.; Aminshahidy, B.; Bazargan, H. Prediction of waterflood performance using a modified capacitance-resistance model: A proxy with a time-correlated model error. J. Pet. Sci. Eng. 2021, 198, 108152.

(23) Yousefi, S. H.; Rashidi, F.; Sharifi, M.; Soroush, M. Prediction of immiscible gas flooding performance: a modified capacitanceresistance model and sensitivity analysis. Pet. Sci. 2019, 16, 10861104.

(24) Tao, Q.; Bryant, S. L. Optimizing CO2 Storage in a Deep Saline Aquifer with the Capacitance-resistance Model. Energy Procedia 2013, 37, 3919-3926.

(25) Eshraghi, S. E.; Rasaei, M. R.; Zendehboudi, S. Optimization of miscible $\mathrm{CO}_{2}$ EOR and storage using heuristic methods combined with capacitance/resistance and Gentil fractional flow models. J. Nat. Gas Sci. Eng. 2016, 32, 304-318.

(26) Yewgat, A.; Busby, D.; Chevalier, M.; Lapeyre, C.; Teste, O. Deep-CRM: A New Deep Learning Approach for Capacitance Resistive Models. ECMORXVII: 17 th European Conference on The Mathematics of Oil Recovery; European Association of Geoscientists \& Engineers: Online, 2020.

(27) Holanda, R.; Gildin, E.; Jensen, J.; Lake, L.; Kabir, C. A State-ofthe-Art Literature Review on Capacitance Resistance Models for Reservoir Characterization and Performance Forecasting. Energies 2018, 11, 3368

(28) Cao, F.; Luo, H.; Lake, L. W. Oil-Rate Forecast by Inferring Fractional-Flow Models from Field Data with Koval Method Combined with the Capacitance/Resistance Model. SPE Reservoir Eval. Eng. 2015, 18, 534-553.

(29) Koval, E. J. A Method for Predicting the Performance of Unstable Miscible Displacement in Heterogeneous Media. Soc. Pet. Eng. J. 1963, 3, 145-154.

(30) Lake, L. W.; Johns, R. T.; Rossen, W. R.; Pope, G. A. Fundamentals of Enhanced Oil Recovery. Society of Petroleum Engineers: 2014.

(31) Mollaei, A.; Delshad, M. General Isothermal Enhanced Oil Recovery and Waterflood Forecasting Model. SPE Annual Technical Conference and Exhibition; Society of Petroleum Engineers: 2011.

(32) Jain, L.; Lake, L. W. Surveillance of Secondary and Tertiary Floods: Application of Koval's Theory to Isothermal Enhanced Oil Recovery Displacement. SPE Improved Oil Recovery Symposium; Society of Petroleum Engineers: 2014.

(33) Farajzadeh, R.; Wassing, B. L.; Lake, L. W. Insights into design of mobility control for chemical enhanced oil recovery. Energy Rep. 2019, 5, 570-578.

(34) Salazar, J. J.; Lake, L. W. The Physical Meaning of the Koval Factor. Math. Geosci. 2020, 52, 1017-1033.

(35) Presser, D. J.; Cafaro, V. G.; Zamarripa, M.; Cafaro, D. C. Optimal Strategies for Carbon Dioxide Enhanced Oil Recovery under Uncertainty. 13th International Symposium on Process Systems Engineering (PSE 2018); Elsevier: 2018, 1507-1512, DOI: 10.1016/B978-0444-64241-7.50246-9.

(36) Yousef, A. A.; Gentil, P. H.; Jensen, J. L.; Lake, L. W. A Capacitance Model To Infer Interwell Connectivity From Production and Injection Rate Fluctuations. SPE Reservoir Eval. Eng. 2006, 9, 630646.

(37) Ibiam, E.; Geiger, S.; Demyanov, V.; Arnold, D. Optimization of Polymer Flooding in a Heterogeneous Reservoir Considering 
Geological and History Matching Uncertainties. SPE Reservoir Eval.

Eng. 2021, 24, 19-36.

(38) Al-Shakry, B.; Shaker Shiran, B.; Skauge, T.; Arne, S. Polymer Injectivity: Influence of Permeability in the Flow of EOR Polymers in Porous Media. SPE Europec featured at 81st EAGE Conference and Exhibition; Society of Petroleum Engineers: 2019.

(39) Dinh, A. V.; Tiab, D. Interpretation of Interwell Connectivity Tests in a Waterflood System. SPE Annual Technical Conference and Exhibition; Society of Petroleum Engineers: 2008.

(40) Zhong, H.; Zhang, W.; Fu, J.; Lu, J.; Yin, H. The Performance of Polymer Flooding in Heterogeneous Type II Reservoirs-An Experimental and Field Investigation. Energies 2017, 10, 454.

(41) Kaviani, D.; Soroush, M.; Jensen, J. L. How accurate are Capacitance Model connectivity estimates? J. Pet. Sci. Eng. 2014, 122, 439-452.

(42) Fanchi, J. R. Principles of Applied Reservoir Simulation; (4th ed.). Gulf Professional Publishing: 2018.

(43) Buckley, S. E.; Leverett, M. C. Mechanism of Fluid Displacement in Sands. Trans. AIME 1942, 146, 107-116.

(44) Moreno, G. A.; Lake, L. W. Input signal design to estimate interwell connectivities in mature fields from the capacitanceresistance model. Pet. Sci. 2014, 11, 563-568.

(45) Ali, M.; Mahmud, H. B. The effects of Concentration and Salinity on Polymer Adsorption Isotherm at Sandstone Rock Surface. IOP Conference Series: Materials Science and Engineering; 2015.

(46) Flory, P. J. Principles of Polymer Chemistry. Amsterdam University Press: 1953.

(47) Wang, J.; Liu, H. A novel model and sensitivity analysis for viscoelastic polymer flooding in offshore oilfield. J. Ind. Eng. Chem. 2014, 20, 656-667.

(48) Manzoor, A. A. Modeling and Simulation of Polymer Flooding with Time-Varying Injection Pressure. ACS Omega 2020, 5, 52585269.

(49) Akbari, S.; Mahmood, S. M.; Ghaedi, H.; Al-Hajri, S. A. New Empirical Model for Viscosity of Sulfonated Polyacrylamide Polymers. Polymer 2019, 11, 1046.

(50) Albertoni, A.; Lake, L. W. Inferring Interwell Connectivity Only From Well-Rate Fluctuations in Waterfloods. SPE Reservoir Eval. Eng. 2003, 6, 6-16.

(51) UTCHEM Technical Documentation Volume II Documentation for UTCHEM 2017_3: A Three-Dimensional Chemical Flood Simulator; UTCHEM Technical Documentation: 2017.

(52) McCarl, B. A.; Meeraus, A.; Van der Eijk, P.; Bussieck, M.; Dirkse, S. Expanded GAMS User Guide Version 23.6. GAMS Development Corporation; 2011.

(53) Grossmann, I.; Viswanathan, J.; Vecchietti, A.; Raman, R.; Kalvelagen, E. GAMS/DICOPT: A discrete continuous optimization package. GAMS Corporation Inc.; 2002, 37, 55.

(54) Cafaro, D. C.; Cerdá, J. Dynamic scheduling of multiproduct pipelines with multiple delivery due dates. Comput. Chem. Eng. 2008, 32, 728-753.

(55) Tavallali, M. S.; Karimi, I. A. Integrated Oil-Field Management: From Well Placement and Planning to Production Scheduling. Ind. Eng. Chem. Res. 2016, 55, 978-994. 